\title{
An immersed boundary method for direct and large eddy simulation of stratified flows in complex geometry
}

\author{
Narsimha R. Rapaka ${ }^{a}$, Sutanu Sarkar ${ }^{\mathrm{a}, *}$ \\ ${ }^{a}$ Mechanical and Aerospace Engineering, University of California, San Diego, La Jolla, CA 92093, USA
}

\begin{abstract}
A sharp-interface Immersed Boundary Method (IBM) is developed to simulate density-stratified turbulent flows in complex geometry using a Cartesian grid. The basic numerical scheme corresponds to a central second-order finite difference method, third-order Runge-Kutta integration in time for the advective terms and an alternating direction implicit (ADI) scheme for the viscous and diffusive terms. The solver developed here allows for both direct numerical simulation (DNS) and large eddy simulation (LES) approaches. Methods to enhance the mass conservation and numerical stability of the solver to simulate high Reynolds number flows are discussed. Convergence with second-order accuracy is demonstrated in flow past a cylinder. The solver is validated against past laboratory or numerical results in flow past a sphere, and in channel flow with and without stratification. Since topographically generated internal waves are believed to result in a substantial fraction of turbulent mixing in the ocean, we are motivated to examine oscillating tidal flow over a triangular obstacle to assess the ability of this computational model to represent nonlinear internal waves and turbulence. Results in laboratory-scale (order of few meters) simulations show that the wave energy flux, mean flow properties and turbulent kinetic energy agree well with our previous results obtained using a body-fitted grid (BFG). The deviation of IBM results from BFG results is found to increase with increasing nonlinearity in the wave field that is associated with either increasing steepness of the topography relative to the internal wave propagation angle or with the amplitude of the oscillatory forcing. LES is performed on a large scale ridge, of the order of few kilometers in length, that has the same geometrical shape and same non-dimensional values for the governing flow and environmental parameters as the laboratory-scale topography, but significantly larger Reynolds number. A non-linear drag law is utilized in the large-scale application to parameterize turbulent losses due to bottom friction at high Reynolds number. The large scale problem exhibits qualitatively similar behavior to the laboratory scale problem with some differences: slightly larger intensification of the boundary flow and somewhat higher non-dimensional values for the energy fluxed away by the internal wave field. The phasing of wave breaking and turbulence exhibits little difference between small-scale and large-scale obstacles as long as the important non-dimensional parameters are kept the same. We conclude that IBM is a viable approach to the simulation of internal waves and turbulence in high Reynolds number stratified flows over topography.
\end{abstract}

(C) 2011 Published by Elsevier Ltd.

Keywords: immersed boundary method, stratified flows, oceanic flows, internal gravity waves, turbulence, DNS, LES.

\section{Introduction}

We discuss an immersed boundary method (IBM) that has been developed to simulate turbulent stratified flows at high Reynolds number over complex, three dimensional topography on a Cartesian grid using a finite difference

\footnotetext{
${ }^{*}$ Corresponding author. Tel.: +1 8585348243 .

Email address: sarkar@ucsd.edu (Sutanu Sarkar)
} 
method. The motivating application is internal tides and associated turbulence near rough underwater topography, although the solver can be used for a variety of environmental and engineering flows that include an active scalar that influences the flow field through buoyancy. For problems of large computational size, the solver employs a domain decomposition method that utilizes the Message Passing Interface (MPI) for parallel processing. At low Reynolds numbers, the flow can be simulated using DNS and LES can be employed at high Reynolds number.

Internal tides are internal gravity waves generated when the barotropic tide oscillates over underwater obstacles in a stratified fluid. Some of the energy converted from the barotropic tide to the baroclinic flow is locally trapped and dissipated due to small-scale turbulence near the topography while the rest is radiated away from the topography. The importance of internal waves at topography stems from observations (Ledwell et al., 2000; St. Laurent et al., 2001; Klymak et al., 2006) of bottom-enhanced turbulence in the deep ocean that is a substantial fraction of turbulent mixing necessary to maintain the observed oceanic stratification Wunsch and Ferrari (2004). Internal tide generation in the ocean involves interaction of the flow with complex, three dimensional rough topography. Nonlinear processes and turbulence during wave generation become important when the slope angle becomes equal to or larger than the internal wave propagation angle. For internal waves impinging on a boundary, the wave Froude number $\left(F r=U / C_{p}\right.$ where $U$ is the fluid velocity and $C_{p}$ is the phase velocity of the internal wave) is an additional parameter that controls the breaking of internal waves (Venayagamoorthy and Fringer, 2007; Chalamalla et al., 2013). The numerical resolution of strongly nonlinear internal waves, overturns in the density field, and turbulence require solution of the threedimensional, Navier Stokes equations without making the hydrostatic assumption that is commonly made in largescale ocean models. We have previously developed a finite difference solver for stratified turbulent flows at laboratory scale and lower Reynolds numbers (Gayen and Sarkar, 2011b) that employs a structured grid, generalized coordinates and a computational domain that conforms to two-dimensional topography. The solver has been applied to laboratory scale models of a continental slope by Gayen and Sarkar (2011b) and a ridge by Rapaka et al. (2013). However, the application of a body-conforming structured grid to high Reynolds number oceanic flows over complex topography is scarce.

Stratified flows present the additional complexity of coupling between velocity and density fields that leads to internal gravity waves, new flow instabilities and buoyancy-affected turbulence. The slope angle of underwater topography need to be accurately represented in the simulation because the local conversion from the oscillating tidal energy to internal gravity wave energy depends substantially on whether the slope angle is less than (subrcitical), equal to (critical), or greater than (supercritical) the wave propagation angle even in the linear case (Llewellyn Smith and Young, 2002; Pétrélis et al., 2006). Turbulence is found to be intensified at near-critical slopes in observations (Moum et al., 2002; Nash et al., 2007) and turbulence-resolving simulations (Gayen and Sarkar, 2011a,b), as well as near supercritical topography as shown by observations (Klymak et al., 2008; Alford et al., 2011) and suggested by two-dimensional simulations (Legg and Klymak, 2008; Klymak et al., 2010). Slope angle is also important when internal waves shoal on to the continental slope as recently reviewed by Lamb (2014) and examined through turbulence-resolving simulations at laboratory scale by Arthur and Fringer (2014).

The MIT GCM (Marshall et al., 1997) is a finite volume, non-hydrostatic model that handles topography with partially filled cells leading to a boundary region with piecewise constant cell thickness. SUNTANS (Fringer et al., 2006) is an unstructured finite volume, non-hydrostatic model. Although unstructured meshes are well-suited to complex geometry, turbulence resolving computations using LES and central difference schemes are not straightforward with this method. Moreover, unstructured meshes are not better in accuracy or cost than structured meshes. Topographic internal wave problems have been simulated with the MIT GCM (Legg and Klymak, 2008; Klymak et al., 2010), and with SUNTANS (Kang and Fringer, 2011; Zhang et al., 2011). More recently, SOMAR (Santilli and Scotti, 2015), a generalized-coordinate, structured grid solver with adaptive mesh resolution (AMR) has been developed for oceanic problems. None of these models, although non-hydrostatic, claim to tackle turbulence in the sense of large eddy simulation (LES) that resolves the energy containing turbulent motions.

Recent advances in the IBM show promise towards the goal of simulating flows in complex geometries while retaining a structured grid, and encourage us to develop the IBM for simulations of stratified flows over complex topography. Due to the nature of the linear systems arising from discretization on structured meshes, accuracy and efficiency can be higher compared to unstructured meshes. Two major categories exist in IBM: diffusive interface methods and sharp interface methods, see Mittal and Iaccarino (2005). Diffusive interface methods employ forcing distributed over a few cells surrounding the immersed boundary while sharp interface methods (eg. Mittal et al. (2008)) enforce the no-slip boundary condition on the immersed boundary surface. The sharp interface methods have proved to 
be particularly well suited for wall-bounded turbulent flows where boundary layer turbulence plays an important role in the overall solution accuracy. There are many variants of immersed boundary methods available in the literature but the applicability of IBM to high-Reynolds number flows in a stratified medium with both topographically generated internal waves and turbulence remains an open question.

Several methods have been proposed to perform reconstruction of the flow near immersed boundary (IB). To compute the momentum forcing required to enforce no-slip conditions at the IB, Fadlun et al. (2000) used linear interpolation along Cartesian directions for cells near the immersed boundary in the fluid region. However, there may be ambiguity in the direction of interpolation for highly curved boundaries. Balaras (2004) proposed a method that leads to a unique interpolation stencil along the wall normal direction through the use of an intermediate node. Roman et al. (2009) simplified the method for obtaining the momentum forcing by removing the provisional step used by Balaras (2004) and directly imposing the reconstructed velocities on the cells close to the IB surface. Mittal et al. (2008) used ghost-cells inside the solid body to satisfy the boundary conditions on the IB.

Conservation of mass is very important to accurately predict flows at high Reynolds number. Mark and van Wachem (2008) excluded face velocities at cells inside the solid body when discretizing the continuity equation for the pressure to ensure no mass flux across the immersed boundary. Kang et al. (2009) defined new velocity components on the faces cut by the IB to accurately compute the mass fluxes using finite volume discretization. However, to reduce the complexity, the Laplacian of pressure was discretized using the conventional finite difference operator. Meyer et al. (2010) modified the finite volume discretization of the Navier-Stokes equations near the immersed boundary to conserve mass and used a momentum exchange approach to enforce the boundary conditions on the IB. One way to satisfy the geometric conservation law and local mass conservation accurately is through the use of a Cartesian cutcell method (Ye et al., 1999; Udaykumar et al., 2001). In this method, the IB cells are reshaped into non-rectangular control-volumes and a finite-volume method is used to ensure strict satisfaction of conservation laws. However, the extension of these methods to 3D problems is very complicated. Seo and Mittal (2011) adopted a cut-cell based approach only for the discretization of Poisson and velocity correction equations to enforce geometric conservation and used the volume/face fraction of the fluid region in evaluating the mass fluxes.

In the present work, the governing equations are discretized on a Cartesian grid which allows the use of efficient geometric multigrid method to solve the Poisson equation. An immersed boundary method is developed to model the effect of the complex topography on the flow field. The present method has a sharp fluid-solid interface and utilizes trilinear interpolation similar to that of Mittal et al. (2008). However, there are some differences in the identification of ghost cells and the reconstruction procedure as will be discussed in section 3. An accurate method to compute the mass fluxes is utilized and extended to the momentum equations as well at no additional computational expense. An active scalar field that is coupled to momentum conservation through buoyancy forcing is also included. Large Eddy Simulation (LES) models are employed for tidal flows over large scale topographies. In engineering applications, the near-wall treatment of high-Re boundary layer turbulence is critical to compute the drag. In the motivating application of internal tide generation, the dominant contribution to turbulent losses is from convective and shear instabilities that are not controlled by viscous boundary layer dynamics. Much of the dissipation occurs in regions of the boundary flow that are detached from the boundary. Therefore, a different near-wall treatment through a nonlinear drag law enables extension of simulation capability to larger scale (order kilometer) topography.

The numerical method is described in section 2. In section 3, details of the IBM are discussed. The near wall treatment to impose wall shear stress for high Reynolds number flows is described in section 4 . In sections 5 and 6 , results of flow past a circular cylinder and a sphere, respectively, are discussed and compared with previous work. Second-order convergence of the IBM is also demonstrated. Sections 7 and 8 are validations of the solver in the problems of unstratified and stratified turbulent channel flow, respectively. In section 9, internal tide generation and turbulence at a laboratory-scale obstacle are considered using the developed IBM solver in the direct numerical simulation (DNS) mode. The baroclinic energy budget and turbulence statistics are compared against the previous DNS studies of Rapaka et al. (2013) and Jalali et al. (2014) which utilized a body conforming grid. In section 10, internal tide generation is studied at a large scale topography using large eddy simulation (LES) along with a nonlinear bottom drag law. 


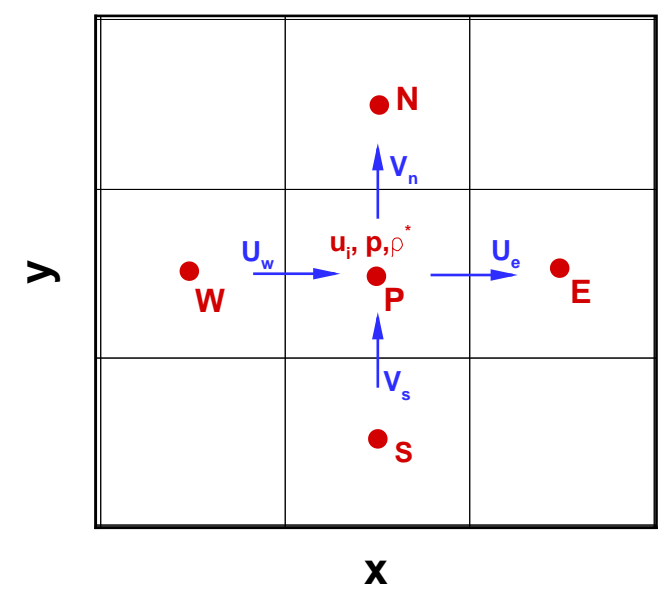

Figure 1: Colocated velocity components $\left(u_{i}\right)$, defined at the cell center P, and face velocity components $\left(U_{i}\right)$, defined at the cell faces $(f=e, w, n, s, t, b)$, are shown. Pressure $(p)$ and density $\left(\rho^{*}\right)$ are colocated with $u_{i}$ at the cell center P.

\section{Numerical method}

Three dimensional Navier-Stokes equations for incompressible flow with the Boussinesq approximation for density coupling are solved numerically on a Cartesian grid. The grid values of velocity, density and pressure are colocated at the center of a notional cell. Face-centered (contravariant) velocities are employed as part of the advective fluxes to advect the grid center variables. Second order central discretization is used for spatial derivatives. A mixed RK3-ADI marching scheme is proposed to advance the solution in time. The diffusion terms are advanced implicitly using an ADI method in three dimensions (Douglas Jr., 1962; Pozrikidis, 2008). All other terms are advanced using an explicit, low-storage Runge-Kutta-Wray3 (RKW3) time marching scheme. The solver allows the use of inhomogeneous boundary conditions in all three directions. We do not include the effect of rotation in the present simulations. But, the inclusion of the Coriolis term in the numerical algorithm to address rotation related physics is straightforward.

$$
\begin{aligned}
\frac{\partial u_{i}}{\partial x_{i}} & =0 \\
\frac{\partial u_{i}}{\partial t}+\frac{\partial\left(U_{j} u_{i}\right)}{\partial x_{j}} & =-\frac{1}{\rho_{0}} \frac{\partial p}{\partial x_{i}}+v \frac{\partial^{2} u_{i}}{\partial x_{j}^{2}}-g \frac{\rho^{*}}{\rho_{0}} \delta_{i 3}-\frac{\partial \tau_{i j}}{\partial x_{j}}, \\
\frac{\partial \rho^{*}}{\partial t}+\frac{\partial\left(U_{j} \rho^{*}\right)}{\partial x_{j}} & =\kappa \frac{\partial^{2} \rho^{*}}{\partial x_{j}^{2}}-u_{i} \frac{\partial \bar{\rho}}{\partial x_{i}} \delta_{i 3}-\frac{\partial \lambda_{j}}{\partial x_{j}},
\end{aligned}
$$

where $u_{i}$ and $p$ are the velocity and pressure colocated at the cell center $(P)$, as shown in fig. 1. $\rho^{*}(x, y, z, t)$ is the density perturbation relative to the background density $\bar{\rho}(z)$ which is computed at the cell center $P$. $U_{i}$ are the face velocity components defined at the cell faces $(f=e, w, n, s, t, b)$ which are offset relative to $P$ by half the cell width in each direction. $U_{i}$ are used in the advection and Poisson source terms to avoid pressure-velocity decoupling. $\tau_{i j}$ and $\lambda_{j}$ are the sub-grid scale stresses for momentum and density equations used in LES. The solver provides the option to estimate the sub-grid scale stresses using the standard Smagorinsky Model (SM) of Deardorff (1970) or the Dynamic Smagorinsky Model (DSM) of Germano et al. (1991). In the present work, SM is used as summarized below,

$$
\tau_{i j}=-2 v_{s g s} S_{i j}, \quad \lambda_{j}=-\kappa_{s g s} \frac{\partial \rho}{\partial x_{j}}, \quad S_{i j}=\frac{1}{2}\left(\frac{\partial u_{i}}{\partial x_{j}}+\frac{\partial u_{j}}{\partial x_{i}}\right),
$$

where the subgrid-scale eddy viscosity and eddy diffusivity are given by

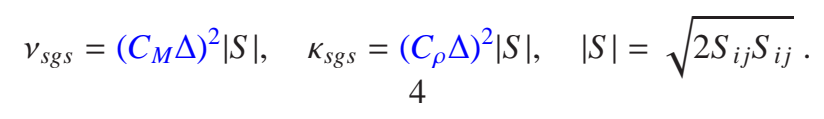


Here, $C_{M}=0.1, C_{\rho}=C_{M} / \operatorname{Pr}_{s g s}$ with a SGS Prandtl number $\left(\operatorname{Pr}_{s g s}\right)$ of unity, and the filter width for the cell $(\Delta)$ is defined by $\Delta \equiv\left(\Delta_{1} \Delta_{2} \Delta_{3}\right)^{\frac{1}{3}}$. The filter width in each direction, $\Delta_{i}$, is taken to be equal to the grid size in that direction. Note that the turbulent Prandtl number $P r_{t}=v_{t} / \kappa_{t}$, which is based on resolved-scale fluxes and mean gradients, is different from $P r_{s g s}$. The value of $P r_{t}$ is not a LES model coefficient, it can differ from unity in stably stratified flows, and it increases with increasing Richardson number, e.g. see fig. 17 of Armenio and Sarkar (2002) and the work of Venayagamoorthy and Stretch (2010).

The mixed RK3-ADI temporal integration is detailed in Appendix A. In each RK3-ADI substep, a predictorcorrector algorithm is used to advance the discretized momentum equation along with continuity equation from step $n$ to $n+1$. The predicted velocity $\left(u^{*}\right)$ is obtained with a guess for the pressure $\left(p^{*}\right)$, typically the pressure at the previous time step $\left(p^{n}\right)$, by solving,

$$
\frac{u_{i}^{*}-u_{i}^{n}}{\Delta t}=-\frac{\delta\left(U_{j} u_{i}\right)^{n}}{\delta x_{j}}+v \frac{\delta}{\delta x_{j}} \frac{\delta u_{i}{ }^{*}}{\delta x_{j}}-\frac{1}{\rho_{0}} \frac{\delta p^{*}}{\delta x_{i}}-g \frac{\rho^{*^{n}}}{\rho_{0}} \delta_{i 3} .
$$

Here, $\frac{\delta}{\delta x_{j}}$ represents the spatial discretization operator. The face velocity components, $U_{i}$, are predicted by using colocated velocity components at neighboring cell centers $(n b=E, W, N, S, T, B$ in fig. 1) separated by the corresponding face $(f)$ (see Zang et al. (1994)). For the face velocities, we first compute an intermediate colocated velocity $\left(\tilde{u}_{i}\right)$ by subtracting the change owing to the pressure gradient,

$$
\tilde{u}_{i} \equiv u_{i, P}^{*}+\left.\frac{\Delta t}{\rho_{0}} \frac{\delta p^{*}}{\delta x_{i}}\right|_{P}
$$

and then, via interpolation, compute the face velocity, $U_{i}^{*}$, as

$$
U_{i}^{*}=\left\{\tilde{u}_{i, P}, \tilde{u}_{i, n b}\right\}-\left.\frac{\Delta t}{\rho_{0}} \frac{\delta p^{*}}{\delta x_{i}}\right|_{f}
$$

Note that the above term adds back the pressure-gradient contribution based on cell face to the intermediate velocity. The terms in the $\{.,$.$\} represents a one-dimensional linear interpolation between the intermediate colocated velocity$ values at $P$ and $n b$. The central difference scheme, used in the present work, provides little numerical diffusion compared to the upwind based schemes, used in Zang et al. (1994), and does not suppress checker board pressure that may arise from pressure-velocity decoupling on a grid with colocated velocity and pressure. Therefore, the pressure gradient is modified, as described in Rhie and Chow (1983), using Eq. (7-8) as a means to address the potential instability from pressure-velocity decoupling.

The velocity is then made divergence free by solving the Poisson equation for pressure correction $\left(p^{\prime}\right)$,

$$
\frac{\delta}{\delta x_{i}} \frac{\delta p^{\prime}}{\delta x_{i}}=\frac{\rho_{0}}{\Delta t} \frac{\delta U_{i}^{*}}{\delta x_{i}}
$$

and applying a correction to the predicted velocity and pressure using,

$$
\begin{aligned}
u_{i}^{n+1} & =u_{i}^{*}-\left.\frac{\Delta t}{\rho_{0}} \frac{\delta p^{\prime}}{\delta x_{i}}\right|_{P} \\
U_{i}^{n+1} & =U_{i}^{*}-\left.\frac{\Delta t}{\rho_{0}} \frac{\delta p^{\prime}}{\delta x_{i}}\right|_{f} \\
p^{n+1} & =p^{*}+p^{\prime}-v \Delta t \frac{\delta}{\delta x_{j}} \frac{\delta p^{\prime}}{\delta x_{j}}
\end{aligned}
$$

Note that the pressure gradient is calculated at cell center, $P$, in Eq. 10 and at face center, $f$, in Eq. 11, which is the key to ensure coupling between velocity and pressure despite both being colocated at the cell center. The last term in Eq. 12 originates from the implicit treatment of the viscous term in Eq. 6. 
(a)

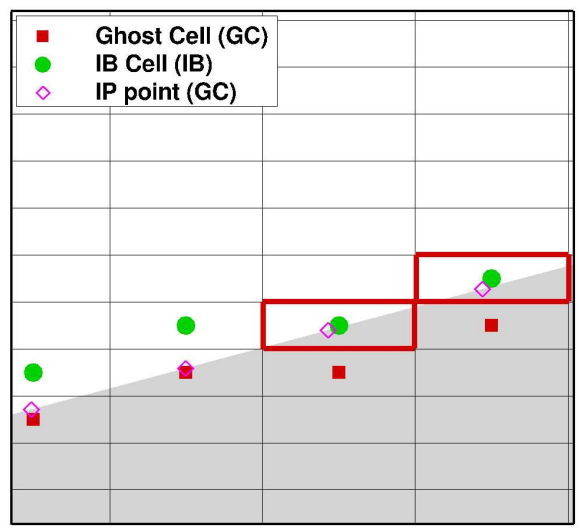

(b)

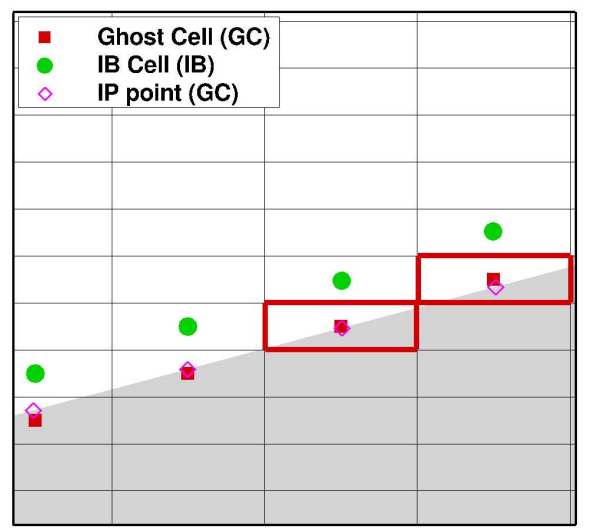

Figure 2: (a) Ghost cells (GC), immersed boundary (IB) cells, and intersection points (IP) on a Cartesian grid distinguished by the immersed body. The shaded area represents the immersed body and the empty boxes are fluid cells. (b) Modified IB cells tagged as GC. The modification leads to more uniform distance between IB cell nodes and the boundary.

\section{Immersed boundary method}

The present IBM is classified as a discrete forcing approach with direct BC imposition (Mittal and Iaccarino, 2005) in which the effect of the immersed boundary is simulated by discretely enforcing the boundary conditions on the immersed boundary surface. The method utilizes a trilinear reconstruction procedure at ghost cells and has a sharp fluid-solid interface similar to that of Mittal et al. (2008). However, there are some differences related to the numerical stability and accuracy of fluxes for DNS and LES of stratified turbulent flows over complex topography. Some key modifications are proposed in the selection of ghost cells, placement of image points, interpolation stencil, and reconstruction procedure as elaborated in the following sections.

\subsection{Geometric preprocessing}

The immersed boundary surface information is provided in a triangulated mesh format and the fluid/solid cells are identified using a ray tracing procedure described in Roman et al. (2009). Figure 2(a) shows a shaded region representing the immersed body, ghost cells (GC), immersed boundary cells (IB) and intersection points (IP). Solid cells that have at least one neighbor, along the Cartesian directions, in the fluid region are tagged as GC. The fluid cells that have atleast one GC as the neighbor along the Cartesian directions are tagged as IB. The IP node for a GC is the intersection of the immersed boundary with the surface normal passing through the center of the corresponding GC. Note that the IP nodes associated with the IB cells are located in a similar way and are not shown in fig. 2(a).

The governing equations are solved at all fluid cells, including the IB cells, while the solution is reconstructed at the GC using trilinear interpolation as described below. The solid cells are excluded from the computational stencil and all variables for the solid cells are explicitly assigned zero values except for pressure. Some of the IB cells, for example highlighted by the two lined boxes in figure 2(a), may have cell centers that lie very close to the immersed boundary. We have observed that the numerical stability of the solver improves if the flow is reconstructed at these cells instead of solving the governing equations especially for high Reynolds number flows. So, if an IB cell is closer to the immersed boundary than the neighbor GC, i.e., $|\overrightarrow{d x}|_{I B-I P}<|\overrightarrow{d x}|_{G C-I P}$ where $|\overrightarrow{d x}|_{I B-I P}$ and $|\overrightarrow{d x}|_{G C-I P}$ are the shortest distances from IB and GC cell centers to the immersed boundary, respectively, then the IB cell is tagged as a GC, as shown in figure 2(b). The original GC is then tagged as a solid cell. The new solid cells are not used in the discretization stencil of any fluid or IB cell, and hence no interpolation is required for these cells. Thus, our method 
(a)

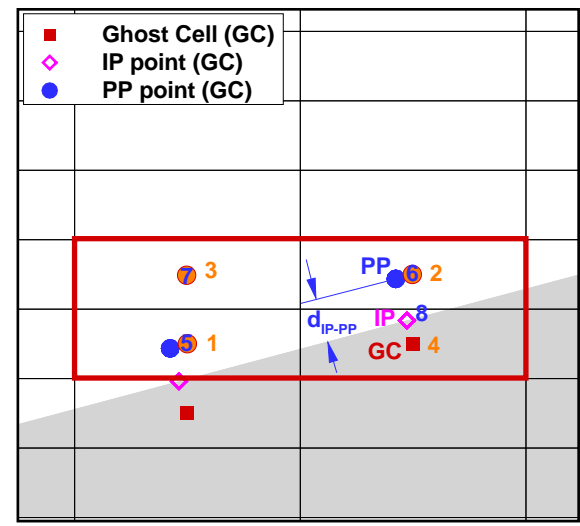

(b)

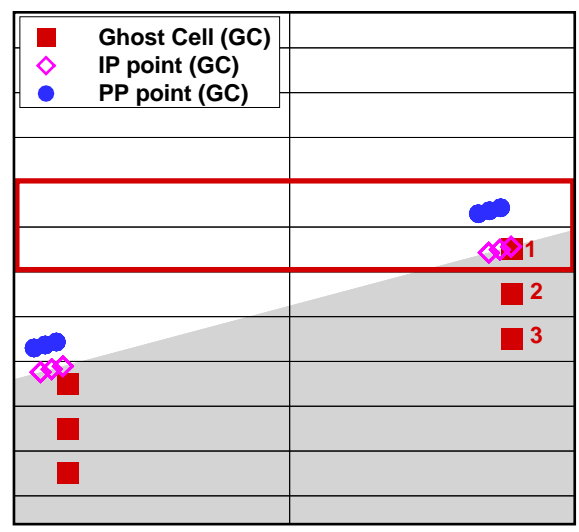

Figure 3: Stencil used in the trilinear interpolation for a GC using a grid with (a) aspect ratio of order 1; Points 5-7 and GC lie in the same plane (say, $S$ ), and points 1-4 lie in a plane parallel $S$; PP is image of the GC along the surface normal connecting IP and GC and is placed closest to a fluid or IB cell center; and (b) large aspect ratio.

has ghost cells on both sides of the immersed boundary and does not restrict them to solid region only (Mittal et al., 2008) or fluid region only (Roman et al., 2009).

\subsection{Reconstruction procedure}

Linear reconstruction is used at GC for all variables, except pressure, at the beginning of each RK3 sub-step. This can be performed in two different ways based on the number of steps used in the reconstruction.

\subsubsection{Direct reconstruction}

The first method, which is described below, consists of a single-step reconstruction and uses trilinear interpolation directly for the GC. The trilinear interpolation is similar to that described in Mittal et al. (2008) but there are few differences in the details which are discussed later. A generic variable $\phi$ is expressed using trilinear interpolation as,

$$
\phi(x, y, z)=C_{1} x+C_{2} y+C_{3} z+C_{4} x y+C_{5} y z+C_{6} x z+C_{7} x y z+C_{8}
$$

The coefficients $C_{i}, i=1,2, \ldots, 8$ are determined using the values of $\phi$ from seven neighbor cells and the boundary condition at the IP node located on the immersed boundary. The neighbors are chosen in all three directions based on the boundary normal direction, as shown in fig. 3(a). The interpolation stencil for a given ghost cell may contain other ghost cells. For instance, consider the interpolation stencil for the labelled ghost cell (GC) indicated by the rectangular box in fig. 3(a). It includes point 4 which is also a ghost cell that lies in a plane parallel to the plane of view and this does not pose any inconsistency. However, for cells with large aspect ratio, e.g., grids used in oceanic flows, more than one GC lie along one of the Cartesian directions as indicated by 1, 2, and 3 in fig. 3(b). If GC 1 , which is close to the IP node of GC2, is included in the interpolation stencil of GC 2, the linear system formed by matrix $[V]$, defined in Eq. 17, may become ill-conditioned and result in spurious values at GC2. To circumvent this issue, the interpolation stencil for all the three $\operatorname{GC}(1,2$, and 3), include cells in the fluid region and the corresponding IP nodes as shown by the red colored rectangular box in fig. 3(b). This interpolation stencil is consistent with that used in the method 2 described later.

Eq. 13 is rearranged into matrix form as,

$$
\{\phi\}=[V]\{C\}
$$


where,

$$
\begin{aligned}
\{C\}^{T} & =\left\{C_{1}, C_{2}, \ldots, C_{8}\right\} \\
\{\phi\}^{T} & =\left\{\phi_{1}, \phi_{2}, \ldots, \phi_{7}, \alpha\right\}
\end{aligned}
$$

and

$$
[V]=\left[\begin{array}{cccccccc}
\left.x\right|_{1} & \left.y\right|_{1} & \left.z\right|_{1} & \left.x y\right|_{1} & \left.y z\right|_{1} & \left.x z\right|_{1} & \left.x y z\right|_{1} & 1 \\
\left.x\right|_{2} & \left.y\right|_{2} & \left.z\right|_{2} & \left.x y\right|_{2} & \left.y z\right|_{2} & \left.x z\right|_{2} & \left.x y z\right|_{2} & 1 \\
\vdots & \vdots & \vdots & \vdots & \vdots & \vdots & \vdots & \vdots \\
\left.x\right|_{7} & \left.y\right|_{7} & \left.z\right|_{7} & \left.x y\right|_{7} & \left.y z\right|_{7} & \left.x z\right|_{7} & \left.x y z\right|_{7} & 1 \\
\left.x\right|_{I P} & \left.y\right|_{I P} & \left.z\right|_{I P} & \left.x y\right|_{I P} & \left.y z\right|_{I P} & \left.x z\right|_{I P} & \left.x y z\right|_{I P} & 1
\end{array}\right]
$$

The last row in Eq.17 assumes Dirichlet boundary condition at IP, that lies on the immersed boundary surface, given by,

$$
\alpha=\phi_{I P}=\left.\left(C_{1} x+C_{2} y+C_{3} z+C_{4} x y+C_{5} y z+C_{6} x z+C_{7} x y z+C_{8}\right)\right|_{I P}
$$

Here, $\alpha$ is the boundary value of $\phi$ specified at IP.

The density (temperature) gradient often satisfies a Neuman boundary condition, e.g., the adiabatic boundary condition employed here. In the case of Neumann boundary condition on the IB surface, the last row of the matrix $[V]$ is modified using,

$$
\begin{aligned}
\alpha & =\left.\nabla \phi \cdot \hat{n}\right|_{I P} \\
& =\left[C_{1} n_{x}+C_{2} n_{y}+C_{3} n_{z}+C_{4}\left(y n_{x}+x n_{y}\right)+C_{5}\left(z n_{y}+y n_{z}\right)\right. \\
& \left.+C_{6}\left(z n_{x}+x n_{z}\right)+C_{7}\left(y z n_{x}+x z n_{y}+x y n_{z}\right)\right]_{I P},
\end{aligned}
$$

where $\alpha$ is the imposed boundary-normal gradient into the fluid and $\hat{n}=n_{x} \hat{\mathbf{i}}+n_{y} \hat{\mathbf{j}}+n_{z} \hat{\mathbf{k}}$ is the unit outward surface normal into the fluid. The above expressions can be simplified when the origin for the interpolation stencil of Eq. 13 is moved to $\vec{x}_{I P}$ so that $\vec{x}=\vec{x}-\vec{x}_{I P}$ and

$$
\begin{aligned}
& \alpha=C_{8} \quad \text { for Dirichlet BC } \\
& \alpha=C_{1} n_{x}+C_{2} n_{y}+C_{3} n_{z} \quad \text { for Neumann BC }
\end{aligned}
$$

The matrix $[V]$ is inverted using the Gauss-Jordan method with full pivoting at each step. The coefficients $\{C\}^{T}$ are then obtained using,

$$
\{C\}=[V]^{-1}\{\phi\} .
$$

At the beginning of each RK3-ADI sub-step, ghost cell values are then evaluated using,

$$
\phi_{G C}=\sum_{i=1}^{8} \beta_{i} \phi_{i}
$$

where

$$
\beta_{i}=v_{G C}^{T}[V]^{-1},
$$

and

$$
v_{G C}^{T}=\left[\begin{array}{llllllll}
\left.x\right|_{G C} & \left.y\right|_{G C} & \left.z\right|_{G C} & \left.x y\right|_{G C} & \left.y z\right|_{G C} & \left.x z\right|_{G C} & \left.x y z\right|_{G C} & 1 .
\end{array}\right]
$$

Note that $\beta_{i}$ include only geometric variables. Hence, this procedure is carried out once at the beginning of the computation and the coefficients $\beta_{i}$ are saved. 


\subsubsection{Reconstruction using an intermediate node (PP)}

The second method is a two-step procedure for interpolation at GC. During the first step, the trilinear interpolation is carried out for the value at the PP node, which is a projection of the GC along the surface normal direction and placed inside a closest fluid or IB cell as shown in fig. 3(a). During the second step, linear interpolation is carried out for GC along the surface normal direction using the PP node and the boundary condition at the IP node as described by Mittal et al. (2008). The ghost cells 1, 2 and 3 shown in fig. 3(b) have their corresponding PP nodes lie within the same fluid cell. Therefore, PP nodes of all three ghost cells have the same interpolation stencil, indicated by the red-colored rectangular box in fig. 3(b). Thus, both the first and second methods have consistent interpolation stencil and give similar results.

In Mittal et al. (2008), PP is placed such that IP is midway between GC and PP along the surface normal direction, i.e., $|\overrightarrow{d x}|_{P P-I P}=|\overrightarrow{d x}|_{G C-I P}$. However, the PP located using this method reflects the non-uniform distribution of the GC. This would lead to non-uniform errors in the vicinity of immersed boundary and, in our experience, can cause numerical instabilities (small-scale, spurious waves) in simulations of high-Reynolds number stratified flows where the density couples strongly to the velocity through the buoyancy term. In the present approach, PP is placed such that it must lie inside a fluid or an IB cell that is closest to the surface normal. This usually places PP at a characteristic distance from IP (i.e., the immersed boundary) of $d_{I P-P P}$ such that $d_{I P-P P}=(0.5-1.5) \min (\Delta x, \Delta y, \Delta z)$ as shown in fig. 3(a-b). Here, we assume that the wall normal grid spacing is small compared to the other directions parallel to the wall. Note that our present method in which the GC lies on both sides of the immersed boundary further reduces the non-uniform distribution of the PP points.

In Mittal et al. (2008), the interpolation stencil for PP node, matrix $[V]$ in Eq. 17, may not neccessarily include the boundary condition at IP, if it is surrounded by all fluid cells. Also, the row position of the boundary condition, if included, in $[V]$ varies from cell to cell and requires a local search procedure. The first method described earlier allows straightforward identification of the interpolation stencil and includes the boundary condition at IP for each $\mathrm{GC}$ at a fixed row (number 8) of matrix $[V]$. Since the boundary condition is included in the matrix $[V]$, it can also be used to compute values of the variable at other locations such as redefined face center (RFC) introduced in section 3.2.3

In the present work, the first method in which the flow is reconstructed at GC directly is used. The solver additionally provides an option to use the second method involving a two-step procedure for reconstruction at GC. In the present work, the PP nodes are used in the present work to impose bottom drag for large scale topography as described in section 4 .

\subsubsection{Improved mass conservation}

Accurate prediction of mass flux is important in the simulation of high Reynolds number flows. Seo and Mittal (2011) adopted a cut-cell based discretization using fluid area fraction of the cut cell faces to enforce geometric conservation. They limit the method only to the pressure Poisson equation (Eq. 9) and the velocity correction equations (Eq. 10). Here, a method to compute the mass fluxes that is more accurate than the standard procedure is discussed and applied to the advective fluxes in the momentum equations as well at no additional computational expense and complexity.

Figure 4 shows the cells cut by the immersed boundary surface. The part of the cut cell faces lying in the fluid region is highlighted by thick green colored lines and corresponding fluid area fraction $\left(a_{f}\right), \mathrm{f}=\mathrm{e}, \mathrm{w}, \mathrm{n}, \mathrm{s}, \mathrm{t}, \mathrm{b}$, is computed as described by Seo and Mittal (2011). The geometric centroid of the corresponding area is denoted as RFC. In Seo and Mittal (2011), $U_{i}$ is obtained by interpolating the neighboring cell center values on to the cell face as described in Eq. 8. Fluid area fraction of the cut cell face is used to proportionately scale down the mass fluxes. However, $U_{i}$ obtained using this method may not always provide the mass flux through the face accurately, even after scaling down the flux using $a_{f}$. For example, consider $U$ at the cuboid cell face marked by the black colored arrow in fig. 4. If the velocity in the fluid region is positive and a no slip boundary condition is applied at the solid boundary, a linear interpolation would result in negative velocity in the solid region. Thus, since the cell face center lies in the solid region, $U$ (calculated using interpolation of cell-center values) would become negative and results in a negative mass flux through that face, even after the flux is scaled by fluid area fraction. However, a positive velocity in the fluid region would require a net positive mass flux through the fluid region of the face marked by the thick green line in fig. 4. 


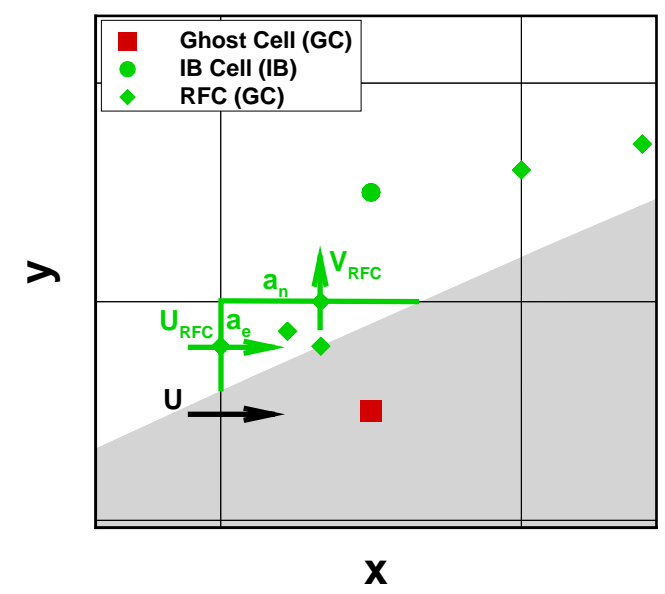

Figure 4: Redefined Face Center (RFC), defined as the geometric centroid of a cut cell face lying in the fluid region indicated by thick green line. The mass fluxes are computed using $U_{i, R F C}$ and corresponding fluid area fraction $a_{f}, f=e, w, n, s, t, w$. The velocity $U_{i}$ at the original cuboid face center is also shown by the black arrow.

In the present method, the mass flux through the fluid region of a cut cell face is evaluated using the face velocity defined at RFC, $U_{i, R F C}$. Since the advective flux through the solid region of a cut cell face is zero, the total advective flux through a cut cell face is given by,

$$
A U_{i}=A_{f} U_{i, R F C}
$$

where $A$ and $A_{f}$ are the total area and the fluid area of each cut cell face and $U_{i, R F C}$ and $U_{i}$ are the velocities at the corresponding face centroids as shown in fig. 4. Noting that the fluid area fraction is given by $a_{f} \equiv A_{f} / A$, the face velocity at the center of the original cuboid cell $\left(U_{i}\right)$ is then given by,

$$
U_{i}=a_{f} U_{i, R F C}
$$

Here, the area fraction is used to accurately compute the mass flux used in both the momentum and the Poisson equations. The face velocities at RFC $\left(U_{i, R F C}\right)$ are evaluated using trilinear interpolation with the interpolation coefficients given by,

$$
\beta_{R F C, i}=v_{R F C}^{T}[V]^{-1}
$$

where, $v_{R F C}^{T}$ is evaluated at the $R F C$ location as,

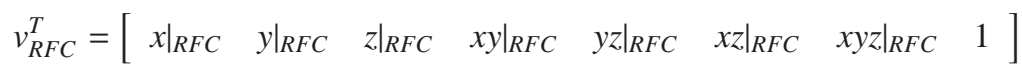

During the predictor step, the face velocities $\left(U_{i, R F C}^{*}\right)$ defined at RFC are computed using,

$$
U_{i, R F C}^{*}=\sum_{k=1}^{8} \beta_{R F C, k} \tilde{u}_{i, k}-\left.\frac{\Delta t}{\rho} \frac{\delta p^{*}}{\delta x_{i}}\right|_{f}
$$

where $\tilde{u}_{i, k}$ are the intermediate colocated velocity components at the neighbor cell centers with pressure gradient subtracted. The predicted face velocity at the original cuboid cell face $\left(U_{i}\right)$ is computed using $U_{i}^{*}=a_{f} U_{i, R F C}^{*}$ which is then used in the Poisson equation, Eq. 9, to solve for the pressure correction. Note that the right hand side of Eq. 9 becomes zero in the solid region as the solid cell faces have zero fluid area fraction. A geometric multi-grid method is used to solve the Poisson equation 9.

Note that the area fraction is not used in the discretized Laplace operator in Eq. 9 since computation of the area fraction at each multi-grid level adds complexity to the implementation of the multi-grid method. Thus, mass 


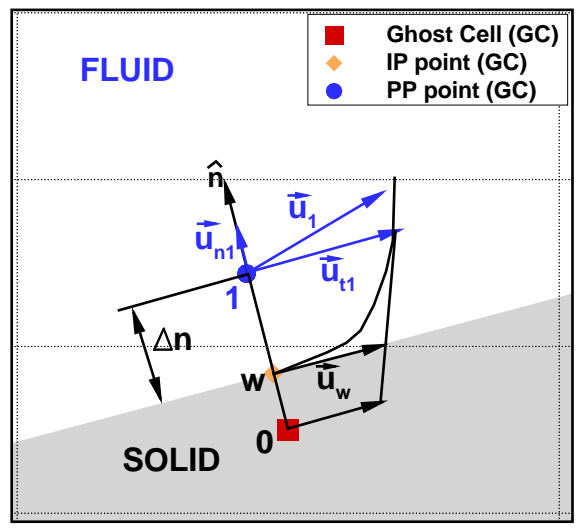

Figure 5: Slip velocity $\left(u_{w}\right)$ that provides the appropriate wall shear stress is imposed at the bottom wall $(w)$. The non-linear drag law based on the tangential velocity $\left(u_{t}\right)$ at point 1 gives the appropriate wall shear stress. Node 1 is the first cell center in a body fitted grid or a PP node in the case of IBM.

conservation is satisfied in an approximate sense similar to the approximate mass conservation described in Kang et al. (2009). Once Eq. 9 is solved in the entire domain, the boundary condition $\frac{\partial p^{\prime}}{\partial n}=0$ is imposed on the immersed boundary, by updating the pressure correction at GC using trilinear interpolation, before the corrector step.

The face velocities for the cut cell faces are then corrected using,

$$
\begin{aligned}
U_{i, R F C}^{n+1} & =U_{i, R F C}^{*}-\left.\frac{\Delta t}{\rho} \frac{\delta p^{\prime}}{\delta x_{i}}\right|_{f} \\
U_{i}^{n+1} & =a_{f} U_{i, R F C}^{n+1}
\end{aligned}
$$

which is then used in computing the advective fluxes in the next time step. The colocated velocity is corrected using,

$$
u_{i}^{n+1}=u_{i}^{*}-\left.\frac{\Delta t}{v_{f} \rho} \frac{\delta\left(a_{f} p^{\prime}\right)}{\delta x_{i}}\right|_{P}
$$

\section{Near wall treatment for large-scale flows}

\subsection{Drag law}

A nonlinear drag law is utilized to model the bottom friction so as to reduce the near wall resolution requirements for higher Reynolds number flows, specifically, over large scale topographies. Let us decompose the velocity into components that are parallel $\left(\overrightarrow{u_{t}}\right)$ and normal $\left(\overrightarrow{u_{n}}\right)$ to the wall so that

$$
\begin{aligned}
\vec{u} & =\overrightarrow{u_{t}}+\overrightarrow{u_{n}} \\
\overrightarrow{u_{n}} & \equiv(\vec{u} \cdot \hat{n}) \hat{n} . \\
& =\left(u n_{x}+v n_{y}+w n_{z}\right) \hat{n}
\end{aligned}
$$

A turbulent surface layer of constant stress is assumed and the first grid point (shown as node 1 in fig. 5 and, hereafter, indicated by subscript 1 ) is taken to lie in the surface layer. Therefore, the shear stress at the no-slip wall $\left(\overrightarrow{\tau_{w}}\right)$ is taken to be equal to the shear stress parallel to the wall at point $1\left(\vec{\tau}_{t 1}\right)$ so that,

$$
\overrightarrow{\tau_{w}}=\vec{\tau}_{t 1}
$$


Here, $\vec{\tau}_{t 1}$ is evaluated at the first cell center above the wall using a non-linear drag model,

$$
\vec{\tau}_{t 1}=\left(\mu_{e} \frac{\partial \vec{u}_{t}}{\partial n}\right)_{1}=\left(C_{D} \rho_{0}\left|\vec{u}_{t}\right| \vec{u}_{t}\right)_{1}
$$

where, $C_{D}$ is the drag coefficient, and $\mu_{e}$ is the effective viscosity, $\mu_{e} \equiv \mu+\mu_{S G S}$, with $\mu_{S G S}$ being the sub-grid scale stress used in LES. The choice of $C_{D}=0.002-0.0025$ is common in oceanographic applications (Legg et al., 2006) and we use $C_{D}=0.0025$ here. It is worth noting that LES of a stratified high-Re bottom boundary layer (Taylor and Sarkar, 2008) with stochastic forcing towards a $\log$ law yielded a value of $C_{D} \simeq 0.0025$. Rearranging the above equation yields,

$$
\left(\frac{\partial \vec{u}_{t}}{\partial n}\right)_{1}=\frac{\left(C_{D} \rho_{0}\left|\vec{u}_{t}\right| \vec{u}_{t}\right)_{1}}{\mu_{e 1}}=\alpha \vec{u}_{t 1} \quad, \quad \alpha=\frac{\left(C_{D} \rho_{0}\left|\vec{u}_{t}\right|\right)_{1}}{\mu_{e_{1}}} .
$$

The boundary condition for the velocity components is then obtained using,

$$
\overrightarrow{\tau_{w}}=\left(\mu_{e} \frac{\partial \vec{u}_{t}}{\partial n}\right)_{w}=\left(\mu_{e} \frac{\partial \vec{u}_{t}}{\partial n}\right)_{1}
$$

so that

$$
\left(\frac{\partial \vec{u}_{t}}{\partial n}\right)_{w}=\frac{\left.\mu_{e}\right|_{1}}{\left.\mu_{e}\right|_{w}}\left(\frac{\partial \vec{u}_{t}}{\partial n}\right)_{1}
$$

At a no-slip wall $\mu_{S G S}=0$ and $\mu_{e}=\mu$, which would result in huge velocity gradients near the wall, $\left(\frac{\partial \vec{u}_{t}}{\partial n}\right)_{w}$. Resolution of the large velocity gradient at the wall and the rapid variation in the inner layer of turbulence is not possible for large-scale oceanic flows. This lack of resolution would result in inappropriate velocities at the near boundary cells and may cause numerical instabilities. To circumvent this problem, Eq. 43 is modified as follows,

$$
\left(\frac{\partial \vec{u}_{t}}{\partial n}\right)_{w}=\left(\frac{\partial \vec{u}_{t}}{\partial n}\right)_{1}=\alpha \vec{u}_{t 1}
$$

that follows from asuuming that $\left.\mu_{e}\right|_{w}=\left.\mu_{e}\right|_{1}$ or $\left(\frac{\partial \mu_{e}}{\partial n}\right)_{w}=0$. Thus a linear discretization of the diffusion fluxes in the momentum equation yields a consistent shear stress and numerical stability. Note that the ghost cell value of $\mu_{S G S}$ is updated using the boundary condition $\frac{\partial \mu_{S G S}}{\partial n}=0$.

\subsection{Boundary conditions for complex topography: slip velocity}

For three dimensional complex topography, the wall boundaries need not be aligned with Cartesian directions. Such cases are simulated using the IBM and they require boundary conditions for the Cartesian velocity components that satisfy the imposed wall shear stress $\left(\left.\frac{\partial \vec{u}_{u}}{\partial n}\right|_{w}\right)$ and the impermeability condition $\left(\left.\vec{u}_{n}\right|_{w}=0\right)$. We prescribe a slip velocity at the wall $\left(\overrightarrow{u_{w}}\right)$ that satisfies the impermeability condition and yields an appropriate velocity gradient, thus shear stress, at the wall. Since $\vec{u}_{t w}$ is parallel to $\vec{u}_{t 1}$, the value of $\vec{u}_{t 1}$ can be expressed in terms of $\vec{u}_{t w}$ using a Taylor series expansion as

$$
\begin{aligned}
\vec{u}_{t 1} & =\vec{u}_{t w}+\left(\frac{\partial \vec{u}_{t}}{\partial n}\right)_{w}(\Delta n) \\
& =\vec{u}_{t w}+\left(\alpha \vec{u}_{t 1}\right)(\Delta n),
\end{aligned}
$$

where $\Delta n$ is the distance from the first cell center to the wall as shown in fig. 5. Rearrangement of Eq. 45 yields

$$
\vec{u}_{t w}=(1-\alpha \Delta n) \vec{u}_{t 1}
$$


Table 1: Quantitative comparison of the flow past a circular cylinder with the literature.

\begin{tabular}{|c|ccc|ccc|ccc|}
\hline $\operatorname{Re}$ & \multicolumn{3}{|c|}{40} & \multicolumn{3}{c|}{100} & \multicolumn{3}{c|}{300} \\
\hline & Henderson & Mittal et al. & present & Henderson & Mittal et al. & present & Henderson & Mittal et al. & present \\
\hline$C_{D f}$ & 0.51 & - & 0.515 & 0.34 & - & 0.326 & 0.20 & - & 0.194 \\
$C_{D p}$ & 1.02 & - & 1.054 & 1.00 & - & 1.035 & 1.17 & - & 1.176 \\
$C_{D}$ & 1.53 & 1.53 & 1.570 & 1.34 & 1.35 & 1.361 & 1.37 & 1.36 & 1.370 \\
$-C_{p b}$ & 0.52 & 0.52 & 0.51 & 0.73 & 0.74 & 0.739 & 1.20 & 1.20 & 1.22 \\
$S t$ & - & - & - & 0.17 & 0.167 & 0.168 & 0.21 & 0.21 & 0.211 \\
\hline
\end{tabular}

Note that the impermeability boundary condition gives $\vec{u}_{n w}=0$ and hence, $\vec{u}_{w}=\vec{u}_{t w}$, allowing the computation of the slip velocity at the wall as

$$
\vec{u}_{w}=(1-\alpha \Delta n) \vec{u}_{t 1}
$$

Note that for inclined surfaces in the IBM, the PP node (an image of the solid region GC node) as shown in figure 5, is used as point 1 where the shear stress is modeled. $\mu_{S G S_{1}}$, used in Eq. 41 , is evaluated at PP nodes using trilinear interpolation including seven neighbor cells and the boundary condition $\frac{\partial \mu_{S G S}}{\partial n}=0$ at the immersed boundary. Eq. 47 is used to prescribe boundary conditions for the Cartesian velocity components at the immersed boundary, which are then used in the reconstruction of the velocity at ghost cells (GC).

\section{Unstratified flow past a circular cylinder at $R e$ up to 300}

In this section, unstratified flow past a circular cylinder is used to validate the solver. As one validation example, we follow several prior authors in performing 2-D simulations at $R e$ up to 300 . The flow remains steady and symmetric up to $R e=47$, beyond which the flow exhibits periodic Karman vortex shedding. The unsteady flow remains twodimensional up to $R e \simeq 180$, beyond which it becomes three-dimensional. The simulations are performed for $R e=$ $U_{\infty} d / v$ up to 300 where $d$ is the cylinder diameter and $U_{\infty}$ is the free stream velocity. A computational grid of $384 \times 384$ is used which is uniform with $\Delta x=\Delta z=0.015 d$ near the cylinder and stretched away from the cylinder. A large domain size of $35 d \times 30 d$ is employed along with sponge at the outflow boundary to prevent reflections from the outflow boundary.

The results including the drag coefficient $C_{D}=F_{D} /\left(\frac{1}{2} \rho U_{\infty}^{2} d\right)$, base pressure coefficient $\left(C_{p b}=\left(p_{b}-p_{\infty}\right) /\left(\frac{1}{2} \rho U_{\infty}^{2}\right)\right)$, and the Strouhal number $\left(S t=f d / U_{\infty}\right)$, where $f$ is the shedding frequency, are shown in table 1. $C_{D f}$ and $C_{D p}$ are the friction and pressure drag coefficients, respectively. For $R e=100$ and 300, the flow becomes unsteady with periodic shedding of vortices. The drag and pressure coefficients are obtained by averaging the corresponding quantities over time after the initial transient. The results agree well with those obtained using a spectral element method (Henderson, 1995) and a immersed boundary method (Mittal et al., 2008).

\subsection{Grid convergence study}

The basic numerical algorithm has second-order spatial truncation error and the trilinear interpolation employed here also has second-order spatial error. To demonstrate the theoretically anticipated second-order convergence of the solver, two-dimensional simulations of flow past a circular cylinder confined between two channel walls are performed at a Reynolds number of $R e=U_{b} d / v=U_{b} \delta / v=100$ where $U_{b}$ is the channel bulk velocity, $\delta$ is the channel halfwidth and $d$ is the cylinder diameter. The simulation is performed on a domain of length $2 d$ in each direction. The flow is then computed on various grids $(70 \times 70,90 \times 90,126 \times 126,210 \times 210$, and $630 \times 630)$ with uniform grid spacing. Since the exact solution for this case is not known, the error at each level is then computed using the solution at the finest grid $(630 \times 630)$ which is well resolved. Note that the center of a given cell on each coarser grid coincides with the center of a cell on the finest grid. This allows the computation of error on coarser grids without any interpolation. The flow is initialized with a parabolic velocity profile and integrated for 2000 time steps with a fixed time step of $0.0001 d / U_{b}$. The error norms computed for streamwise and vertical velocity are shown in fig. $6\left(\right.$ a). Both the $L_{m a x}$ and $L_{2}$ norms in the streamwise and vertical velocity demonstrate second-order convergence. While the $L_{2}$ norm represents global accuracy, the $L_{\max }$ norm represents local error which is maximum near the immersed boundary as shown in fig. 6(b) for the streamwise velocity, $u$, computed on a $126 \times 126$ grid. 
(a)

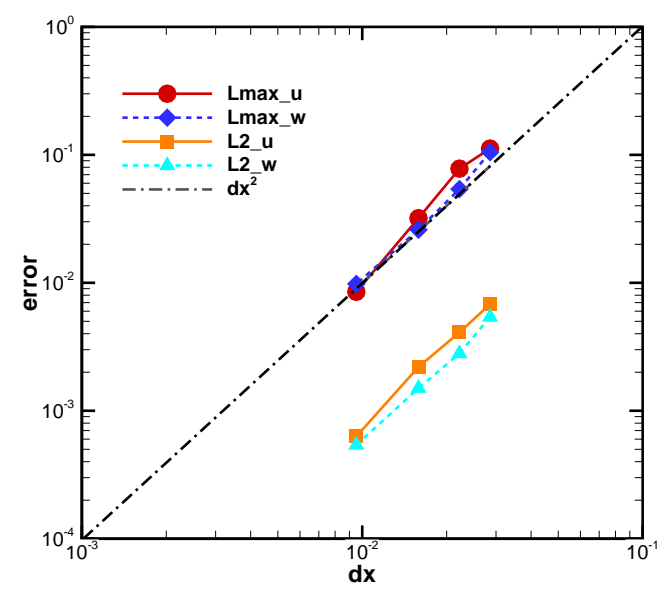

(b)

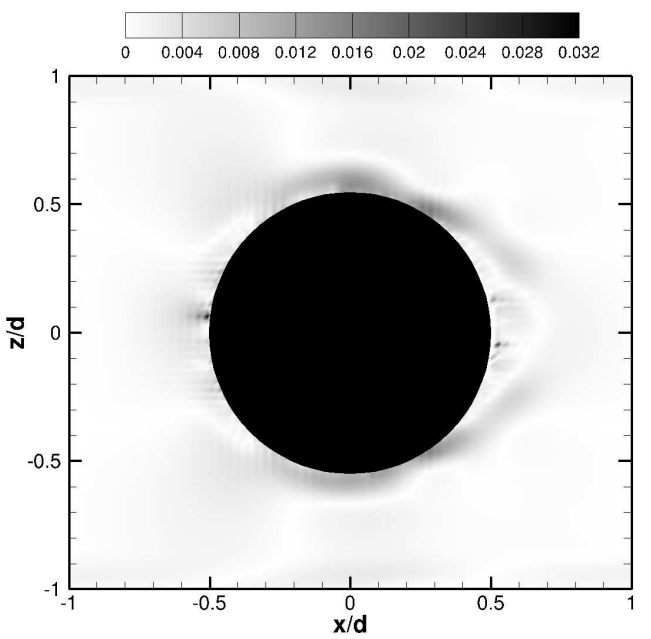

Figure 6: (a) Error norms in streamwise (u) and vertical (w) velocity components verses the grid spacing for flow over a circular cylinder confined between channel walls. (b) Error in the streamwise velocity computed on a $126 \times 126$ grid.

\section{Unstratified flow past a sphere at $R e$ up to 300}

Here we consider unstratified flow past a sphere with increasing Reynolds number up to 300 . The flow is axisymmetric up to $R e \approx 200$, loses axisymmetry and forms streamwise vortices behind the sphere from $210<\operatorname{Re}<270$ and then begins to shed vortices with a constant strength at a consistent frequency for $280<R e<375$.

Data from Johnson and Patel (1999) is available for comparison for $R e \leq 300$. As shown in Figure 7 the flow evolves in a qualitatively similar way between the two cases with both cases showing symmetry about the vertical axis and similar shapes for the recirculating region. Flow statistics such as the separation angle, $\Theta_{s}$, the separation length, $L_{s}$, and the relative position of the vortex core with respect to the center of sphere $\left(X_{c}\right.$ and $\left.Y_{c}\right)$ are calculated and shown in Table 2 for a quantitative comparison in the steady, axisymmetric flow regime. The IBM results agree well with those of (Johnson and Patel, 1999).

\begin{tabular}{ccccccccc}
\hline$R e$ & \multicolumn{2}{c}{$\Theta_{s}$} & \multicolumn{2}{c}{$L_{s} / D$} & \multicolumn{2}{c}{$X_{c} / D$} & \multicolumn{2}{c}{$Y_{c} / D$} \\
\hline & JP & Present & JP & Present & JP & Present & JP & Present \\
\hline 50 & 139 & 139 & 0.42 & 0.40 & 0.64 & 0.64 & 0.21 & 0.20 \\
100 & 127 & 129 & 0.88 & 0.85 & 0.76 & 0.75 & 0.29 & 0.28 \\
150 & 121 & 125 & 1.22 & 1.16 & 0.83 & 0.83 & 0.33 & 0.32 \\
200 & 117 & 120 & 1.46 & 1.39 & 0.88 & 0.87 & 0.36 & 0.34
\end{tabular}

Table 2: Flow past a sphere: comparison with the $R e<200$ results of Johnson and Patel (1999).

In the unsteady planar symmetric regime the flow oscillates at a periodic shedding frequency. This behavior is captured by the present solver. The present simulations led to a Strouhal frequency of 0.133 which compares well with the range $S t=0.134-0.137$ found by Johnson and Patel (1999).

\section{DNS of turbulent channel flow at $\operatorname{Re}_{\tau}=395$}

Turbulent channel flow is simulated at a Reynolds number $R e_{\tau}=u_{\tau} \delta / v=395$ based on half the channel height $(\delta)$ and friction velocity $\left(u_{\tau}\right)$. The IBM method is used to model the solid walls, i.e., the wall is placed in between grid lines. Domain and grid sizes employed in the simulation are $2 \pi \delta \times \pi \delta \times 2 \delta$ and $256 \times 192 \times 192$ in the streamwise 

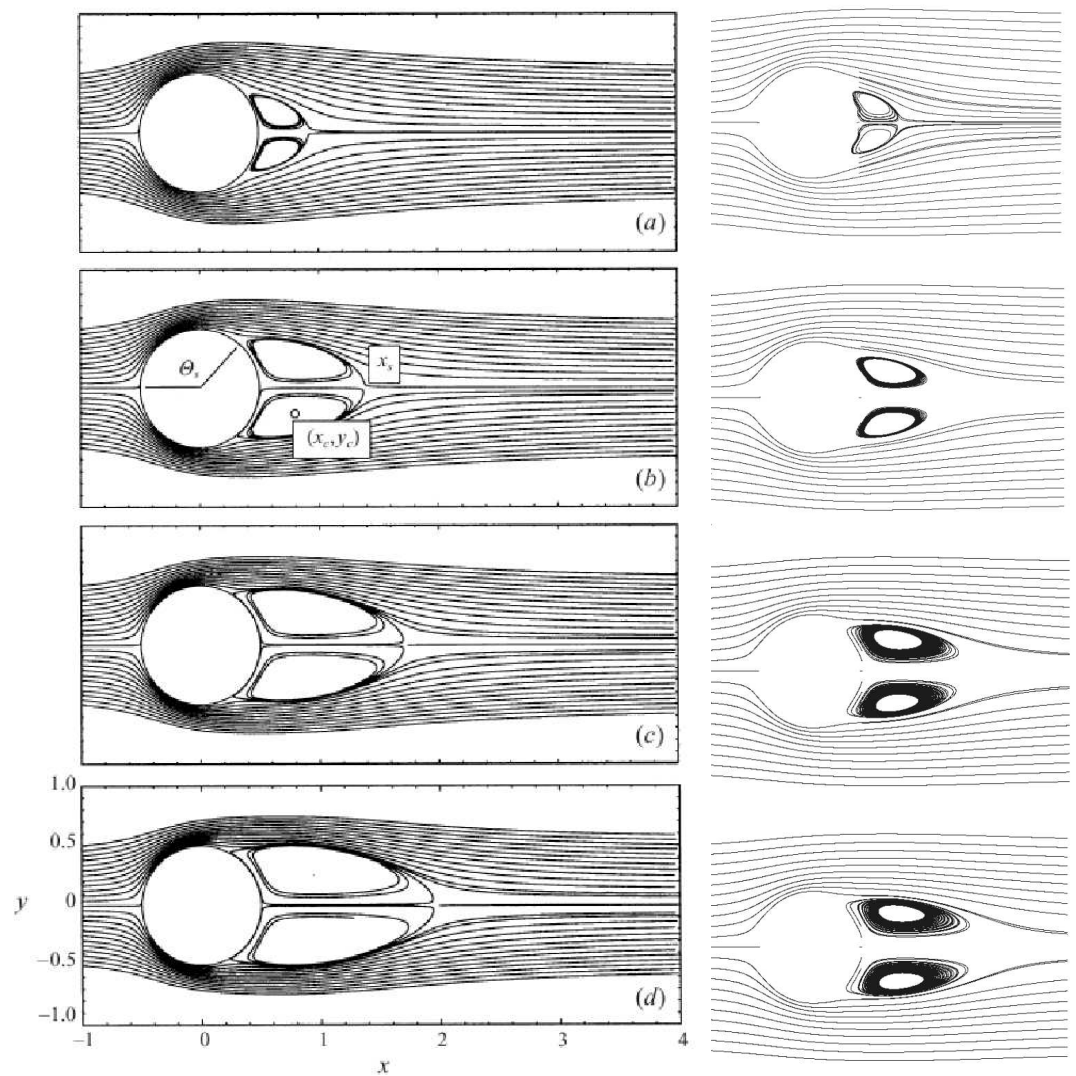

Figure 7: Qualitative comparison of streamlines with Johnson and Patel (1999). (Left column) Data from Johnson and Patel (1999). (Right column) Data from present. From top to bottom: (a) $R e=50$. (b) $R e=100$. (c) $R e=150$. (d) $R e=200$.

$(\mathrm{x})$, the spanwise (y), and the vertical (z) directions, respectively. The grid is uniform in the streamwise and spanwise directions and stretched in the vertical direction from the wall towards the center with resolution in wall units of $\Delta x^{+}=9.69, \Delta y^{+}=6.46, \Delta z_{\text {min }}^{+}=0.59, \Delta z_{\text {max }}^{+}=8.57$. Periodic boundary conditions are employed for all variables in the $\mathrm{x}$ and the $\mathrm{y}$ directions while the no-slip boundary condition $\left(u, v, w=0, d p^{\prime} / d z=0\right)$ is employed on the channel walls $(z= \pm 1)$. The flow is driven by a constant pressure gradient. The initial flow field corresponds to the laminar fully developed flow in a channel superimposed with perturbations obtained using the method of Rogallo (1981).

The turbulence statistics, obtained by averaging along $\mathrm{x}$, y directions and in time, including the Reynolds stress components and the mean velocity are shown in figure 8 (a) and (b), respectively. The profiles agree well with those of Moser et al. (1999).

\section{DNS of stratified turbulent channel flow}

Direct numerical simulation of stratified channel flow is performed at a Reynolds number, $\operatorname{Re}_{\tau}=u_{\tau} \delta / v=180$, based on half the channel height $(\delta)$ and friction velocity $\left(u_{\tau}\right)$ and a friction Richardson number of $R i_{\tau}=18$. Here, $R i_{\tau}=\Delta \rho g \delta / \rho_{0} u_{\tau}^{2}$ where $\Delta \rho$ is the density difference between top and bottom walls. The channel walls are discretized using a triangulated surface mesh and IBM is used to simulate the effect of channel walls. Domain and grid sizes employed in the simulation are $4 \pi \delta \times 4 \pi / 3 \delta \times 2 \delta$ and $128 \times 128 \times 128$ in the streamwise (x), the spanwise (y), and the vertical ( $\mathrm{z}$ ) directions, respectively. The grid is uniform in the streamwise and spanwise directions and stretched in the vertical direction from the wall towards the center with $\Delta x^{+}=19.9, \Delta y^{+}=6.6, \Delta z_{\min }^{+}=0.48, \Delta z_{\text {max }}^{+}=5.9$. Periodic boundary conditions are employed for all variables in the $\mathrm{x}$ and the $\mathrm{y}$ directions while the no-slip boundary 
(a)

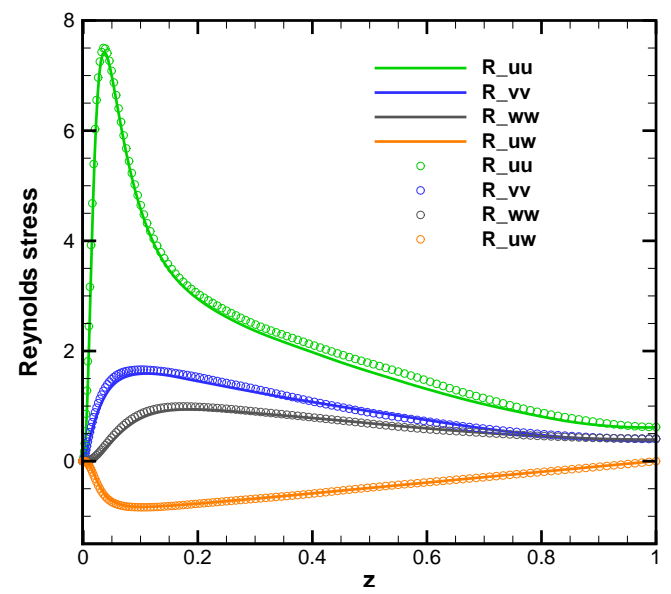

(b)

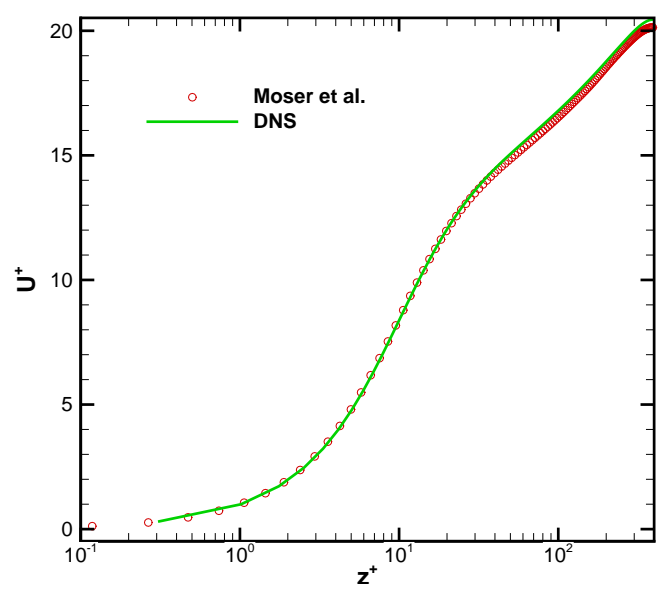

Figure 8: Statistics of unstratified channel flow at $R e_{\tau}=395$, with channel walls simulated using IBM on a grid of 256x192x192: (a) Reynolds stress components, and (b) mean velocity normalized by wall units. Lines correspond to the present DNS results and circles to those of Moser et al. (1999).

condition $\left(u, v, w=0, d p^{\prime} / d z=0\right)$ is employed on the channel walls $(z= \pm 1)$. The flow is driven by a constant pressure gradient. Initial flow field corresponds to the laminar fully developed flow in a channel that is superimposed with perturbations obtained using the method of Rogallo (1981).

The turbulence statistics, obtained by averaging along $\mathrm{x}$, y directions and in time, including the mean velocity and Reynolds shear stress, normalized by wall units, are shown in figure 9 (a) and (b), respectively. The profiles agree well with the numerical studies of Armenio and Sarkar (2002) and Garcia-Villalba and del Álamo (2011).

\section{DNS of stratified flow past a laboratory scale model ridge at $\operatorname{Re}_{s}=177$}

Oscillating flow over a laboratory-scale smoothed triangular ridge (fig. 10) in a stratified environment is studied numerically using the IBM. The flow is driven by an oscillating pressure gradient, $d p / d x=U_{0} \Omega \cos (\Omega t)$, with frequency $\Omega$ that results in an oscillating barotropic velocity of amplitude $U_{0}$. This oscillatory tidal forcing leads to internal waves launched from the ridge as well as near-field turbulence. Internal wave and turbulence properties change qualitatively, depending on the choice of parameters. Therefore, several cases are simulated to explore the ability of IBM in different dynamical regimes. The laboratory scale model (length of order several meters) results are compared to earlier studies of Rapaka et al. (2013) and Jalali et al. (2014) that using a body fitted grid. After successful validation against the body-fitted grid results, large scale simulations (length of the order of several kilometers) are performed to illustrate the capabilities of IBM. Some important non-dimensional parameters that characterize the flow are Reynolds number, $R e_{s}=U_{0} \delta_{s} / v$, based on the Stokes boundary layer thickness $\left(\delta_{s}=\sqrt{2 v / \Omega}\right)$ and the barotropic velocity $\left(U_{0}\right)$; Excursion number, $E x=U_{\infty} / \Omega l$, the ratio of fluid excursion length $\left(l_{e x}=U_{0} / \Omega\right)$ to the topographic length scale $(l)$; and Criticality parameter $(\epsilon=\tan \beta / \tan \alpha)$, the ratio of the topographic slope $(\tan \beta)$ to the internal wave characteristic slope $(\tan \alpha)$. The Reynolds number, $R e=U_{\infty} l_{e x} / v$, based on the fluid excursion length is also of interest.

The cases simulated are listed along with the nondimensional and dimensional parameters in tables 3 and 4 , respectively. The cases marked with IBM and BFG, in the remarks column of table 3 , are simulations using IBM and body fitted grid (BFG), respectively. Cases corresponding to the DNS of a laboratory scale topography are marked by DNS. Cases corresponding to LES of flow past large scale topography are marked by LES and BD indicates that the bottom drag law is used for that simulation.

Two values of excursion number (a measure of nonlinearity of the oscillating tidal forcing) are considered: $E x=$ 0.066 (case numbers starting with 1 ) and $E x=0.4$ (case numbers starting with 2 ). The model ridge has a linear slope 
(a)

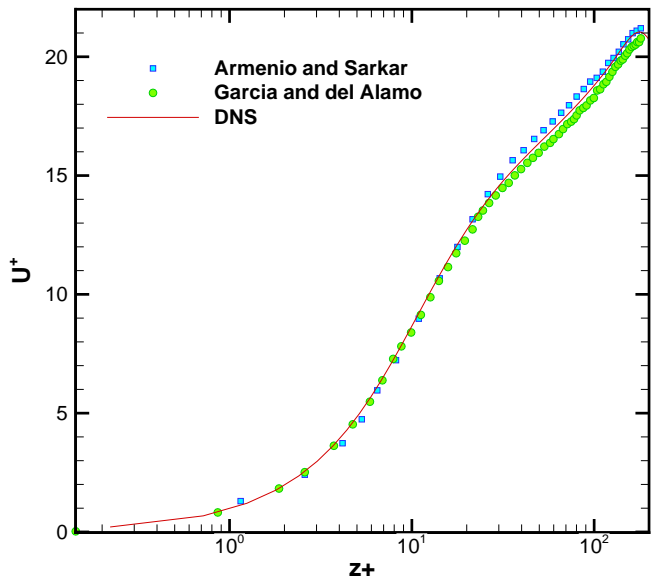

(b)

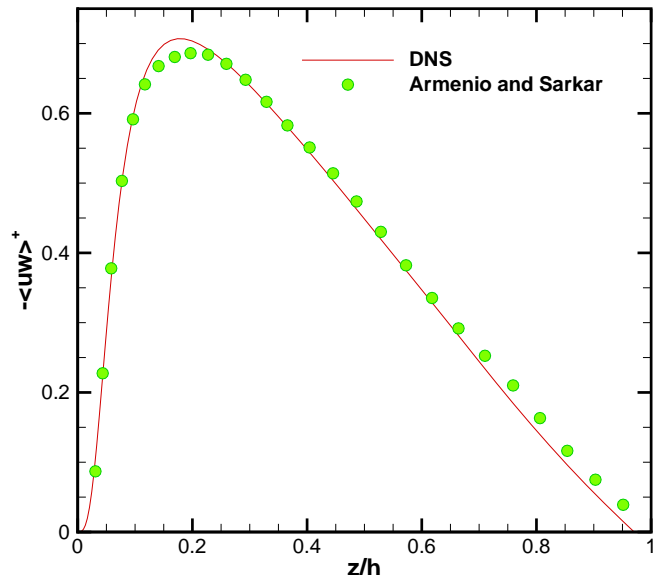

Figure 9: Statistics of stratified channel flow at $R e_{\tau}=180$ and $R i_{\tau}=18$ : (a) mean velocity, and (b) Reynolds shear stress normalized by wall units.

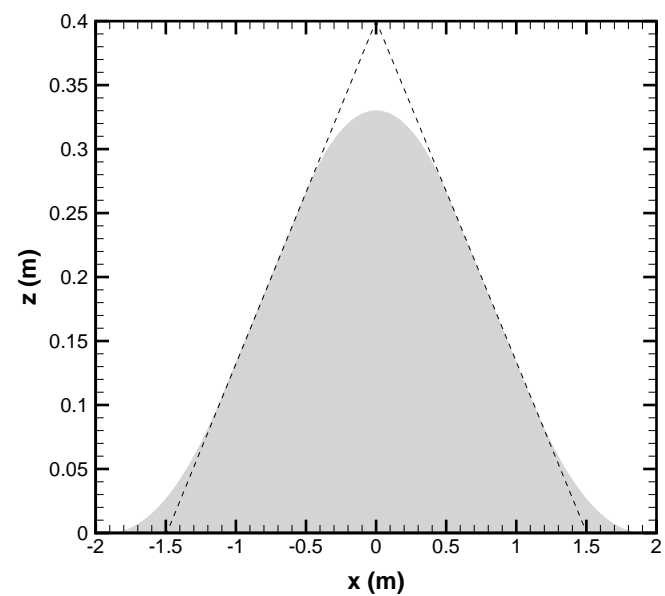

Figure 10: Topography of a smoothed triangular ridge used in laboratory scale simulations, shown by the shaded area. The large scale topography (cases 1cri_ls and 2cri_ls in table 4) use the same shape but the length in each direction is increased by 1000 times. 


\begin{tabular}{lrlcccccccccc} 
case & $R e_{s}$ & $R e$ & $E x$ & $\epsilon$ & $N_{x}$ & $N_{y}$ & $N_{z}$ & $\Delta x_{c}(m)$ & $\Delta x_{M S}(m)$ & $\Delta y(m)$ & $\Delta z_{\min }(m)$ & \multicolumn{1}{c}{$\operatorname{Remark}$} \\
\hline 1sub & 177 & $1.56 \times 10^{4}$ & 0.066 & 0.57 & 896 & 128 & 768 & .006 & .006 & .002 & $6.0 \times 10^{-4}$ & IBM, DNS \\
1 cri & 177 & $1.56 \times 10^{4}$ & 0.066 & 1 & 896 & 128 & 768 & .006 & .006 & .002 & $6.0 \times 10^{-4}$ & IBM, DNS \\
1sup & 177 & $1.56 \times 10^{4}$ & 0.066 & 2.17 & 896 & 128 & 768 & .006 & .006 & .002 & $6.0 \times 10^{-4}$ & IBM, DNS \\
1sub_bf & 177 & $1.56 \times 10^{4}$ & 0.066 & 0.57 & 897 & 256 & 321 & .0026 & .0075 & .002 & $5.4 \times 10^{-4}$ & BFG, DNS \\
1cri_bf & 177 & $1.56 \times 10^{4}$ & 0.066 & 1 & 897 & 256 & 321 & .0026 & .0075 & .002 & $5.4 \times 10^{-4}$ & BFG, DNS \\
1 sup_bf & 177 & $1.56 \times 10^{4}$ & 0.066 & 2.17 & 705 & 128 & 385 & .0125 & .016 & .004 & $4.4 \times 10^{-4}$ & BFG, DNS \\
1cri_ls & 2092 & $2.19 \times 10^{6}$ & 0.066 & 1 & 896 & 64 & 384 & 6.0 & 6.0 & 3.9 & 1.5 & IBM, BD, LES \\
2cri & 177 & $1.56 \times 10^{4}$ & 0.400 & 1 & 896 & 128 & 768 & .006 & .006 & .002 & $6.0 \times 10^{-4}$ & IBM, DNS \\
2cri_bf & 177 & $1.56 \times 10^{4}$ & 0.400 & 1 & 897 & 128 & 321 & .0026 & .0075 & .002 & $5.4 \times 10^{-4}$ & BFG, DNS \\
2cri_ls & 12669 & $8.02 \times 10^{7}$ & 0.400 & 1 & 896 & 64 & 384 & 6.0 & 6.0 & 3.9 & 1.5 & IBM, BD, LES
\end{tabular}

Table 3: Nondimensional parameters and grid spacing of the cases of flow past a ridge. The selection of parameters is discussed in the accompanying text.

\begin{tabular}{lclrrrrrrr} 
case & $U_{0}\left(\mathrm{~ms}^{-1}\right)$ & $N_{\infty}^{2}\left(\mathrm{~s}^{-2}\right)$ & $\Omega\left(s^{-1}\right)$ & $v\left(\mathrm{~m}^{2} \mathrm{~s}^{-1}\right)$ & $l(m)$ & $h(m)$ & $L_{x}(m)$ & $L_{y}(\mathrm{~m})$ & $L_{z}(\mathrm{~m})$ \\
\hline 1 sub & 0.125 & 5.60 & 1 & $10^{-6}$ & 1.9 & 0.328 & 40 & 0.25 & 3.28 \\
1 cri & 0.125 & 14.93 & 1 & $10^{-6}$ & 1.9 & 0.328 & 40 & 0.25 & 3.28 \\
1 sup & 0.125 & 67.33 & 1 & $10^{-6}$ & 1.9 & 0.328 & 40 & 0.25 & 3.28 \\
1sub_bf & 0.125 & 5.60 & 1 & $10^{-6}$ & 1.9 & 0.328 & 40 & 0.5 & 3.28 \\
1 cri_bf & 0.125 & 14.93 & 1 & $10^{-6}$ & 1.9 & 0.328 & 40 & 0.5 & 3.28 \\
1 sup_bf & 0.125 & 67.33 & 1 & $10^{-6}$ & 1.9 & 0.328 & 40 & 0.5 & 3.28 \\
1cri_ls & 0.0175 & $29.26 \times 10^{-8}$ & $1.4 \times 10^{-4}$ & $10^{-6}$ & 1900 & 328 & 40000 & 250 & 3280 \\
2cri & 0.760 & 14.93 & 1 & $3.7 \times 10^{-5}$ & 1.9 & 0.328 & 40 & 0.25 & 3.28 \\
2cri_bf & 0.760 & 14.93 & 1 & $3.7 \times 10^{-5}$ & 1.9 & 0.328 & 40 & 0.25 & 3.28 \\
2cri_ls & 0.106 & $29.26 \times 10^{-8}$ & $1.4 \times 10^{-4}$ & $10^{-6}$ & 1900 & 328 & 40000 & 250 & 3280
\end{tabular}

Table 4: Dimensional parameters of the simulated cases.

with angle $\beta=15^{\circ}$ that is $20 \%$ of its streamwise extent. The stratification sets the buoyancy frequency, $N_{\infty}$, of the background and, thus, the wave propagation angle, $\alpha$. The value of $N_{\infty}$ is varied among the $E x=0.066$ cases to change $\alpha$ so that the linear slope is subcritical $(\beta<\alpha$ or $\epsilon<1)$, critical $(\beta=\alpha$ or $\epsilon=1)$, or supercritical $(\beta>\alpha$ or $\epsilon>1$ ). The internal wave response is expected to be increasingly nonlinear when $\epsilon$ is near unity (the case of resonant response) and also in supercritical cases when $\epsilon$ is much larger than unity. Cases 1cri_bf and 1sup_bf correspond to cases 5 and 5sup of Rapaka et al. (2013), respectively, and case 2cri_bf corresponds to case CEX3 of Jalali et al. (2014). The results of those recent BFG studies are compared with the results obtained here with IBM. Specifically, case 1 sub is compared to case 1sub_bf , case 1sup to 1sup_bf , and case 2cri to case 2cri_bf .

Cases 1cri_ls and 2cri_ls correspond to near critical flows at low and high Ex, respectively, over a large scale model ridge with shape same as in fig. 10 but length in each direction is increased by 1000 times. Note that these two cases have the same nondimensional parameters (see table 3) as those of cases 1cri and 2cri, respectively, except that they have much larger Reynolds numbers $\left(R e_{s}, R e_{l}\right)$. The results of these two cases are summarized later in section 10.

The domain is $L_{x} \mathrm{~m} \times L_{y} \mathrm{~m} \times L_{z} \mathrm{~m}$ in the streamwise (x), the spanwise (y), and the wall normal (z) directions, respectively. Sponge region is employed at both the boundaries in the streamwise $(x)$ direction to absorb the outgoing internal waves. Periodic boundary conditions are employed for all variables in the spanwise (y) direction. The no-slip condition $(u, v, w=0)$ and $d p^{\prime} / d z=0$ are applied at the bottom boundary $(z=h(x))$ while the rigid lid boundary condition $\left(w=0, d / d z\left(u, v, p^{\prime}\right)=0\right)$ is employed at the top boundary $(z=H)$.

The IBM cases use uniform grid over the topography in order to resolve the boundary layer throughout the height of the topography. The grid is stretched away from the topography with up to a maximum of $4 \%$ and $3 \%$ stretching in the horizontal and vertical directions, respectively. Even if the number of grid points is same, the IBM has lower boundary-normal resolution compared to the body fitted grid, because some of the grid points lie inside the solid region. Additional points are used in the $z$ direction for IBM cases to make the grid resolution comparable to the BFG. These additional points provide better resolution in the region away from the immersed body when compared to the 
(a)

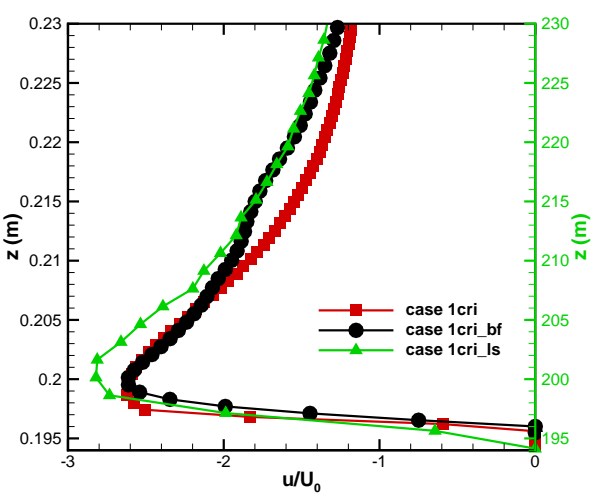

(b)

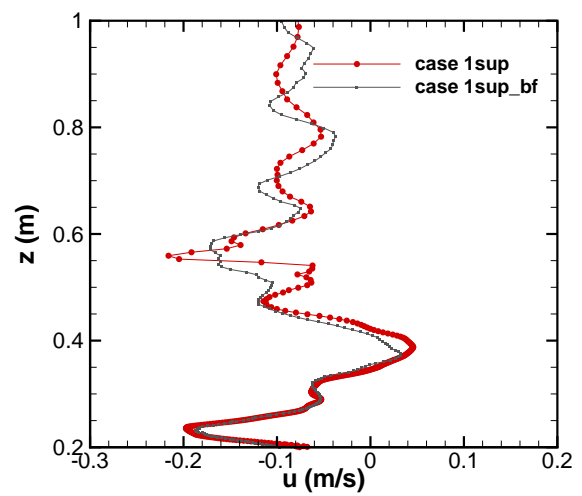

Figure 11: Mean velocity on the slope. Vertical profiles of the streamwise mean velocity at the left mid slope $(x / l=$ -0.405): (a) case 1cri shown at a phase of peak downslope velocity; and (b) case 1sup shown at t/T $=7.59$. Here, $\mathrm{T}$ is the barotropic cycle time period. Note that the z-axis for case 1cri_ls is shown on the right side of figure (a), and, if normalized by a factor of 1000 , collapses onto that of case $1 \mathrm{cri}$.

BFG. This is useful in problems where important nonlinear flow features are present away from the boundary, e.g., internal tide generation at large $E x$ and wakes generated behind a bluff body. Also, note that the ghost cells used in the IBM have a distribution near the immersed boundary that is non-uniform relative to the BFG which also affects the flow resolution.

\subsection{Mean velocity on the slope}

Figure 11(a) shows the vertical profiles of the normalized streamwise mean velocity, $\langle u\rangle_{y}$, corresponding to the phase of peak downslope velocity at the left midslope $(x / l=-0.405)$ for cases 1 cri, 1 cri_bf , and 1 cri_ls . The intensification of the near boundary velocity (almost a factor of 3 larger than the oscillatory forcing velocity) is present in the IBM simulation. The downward propagating boundary flow has thickness and amplitude in case $1 \mathrm{cri}$, using IBM, that are similar to those in 1cri_bf . However, there is a small difference in the boundary layer, likely because case 1 cri has lower wall-normal resolution compared to case 1cri_bf . Also, the ghost cells (and the IB fluid cells adjacent to the boundary) have a distribution that is not as uniform as the near-wall cells in the BFG, which also leads to differences in the boundary layer velocity profiles at the slope.

Figure 11(b) compares cases 1sup, and 1sup_bf, corresponding to a non-dimensional time, $t / T$, of 7.59. Case 1sup shows approximately the same magnitude and shape as that of case 1sup_bf in the core of the beam. Away from the topography, the IBM case has a coarser grid that results in some differences in the baroclinic response above $z=0.4 m$. These profiles suggest that, given similar resolution, IBM yields similar velocity field as that using a body fitted grid.

\subsection{Baroclinic response}

The amount of energy converted from the oscillatory forcing (barotropic tide) to internal waves (the internal tide) and the resultant energy fluxed by the internal waves is a is a result of key interest because this is the energy that could result in eventual turbulent mixing. To study the energetics of the baroclinic response, the spanwise mean velocity and pressure $\left(<u>_{y}(x, z, t),<p>_{y}(x, z, t)\right)$ are decomposed into barotropic and baroclinic components. The barotropic components are computed as the depth average of the spanwise mean which, then, depend only on $x$ and $t$. The baroclinic component, which, by definition, has a zero mean along the depth and also over the tidal cycle, is computed from Eq. 48. See appendix A of Rapaka et al. (2013) for more details.

$$
<\phi>_{y}(x, z, t)-<\phi>_{y T}(x, z)=\phi_{b t}(x, t)+\phi_{b c}(x, z, t),
$$


(a)

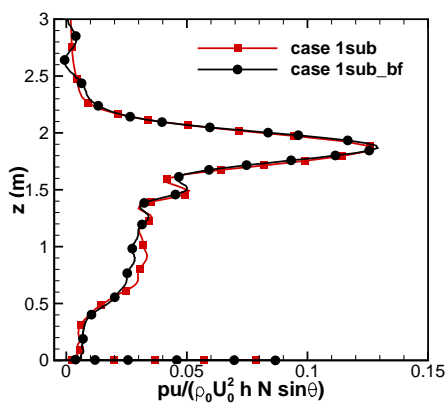

(b)

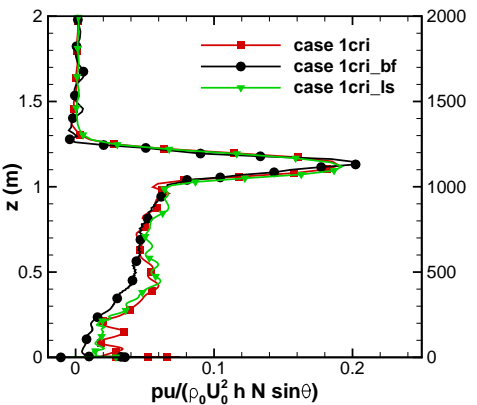

(d)

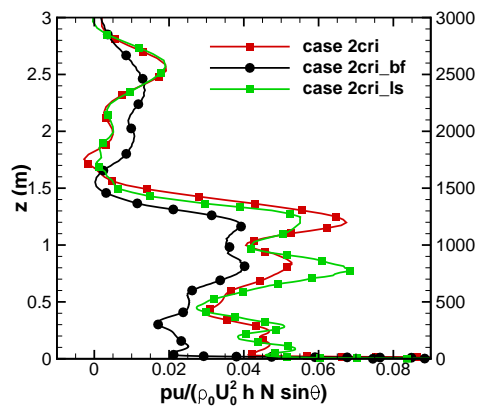

(c)

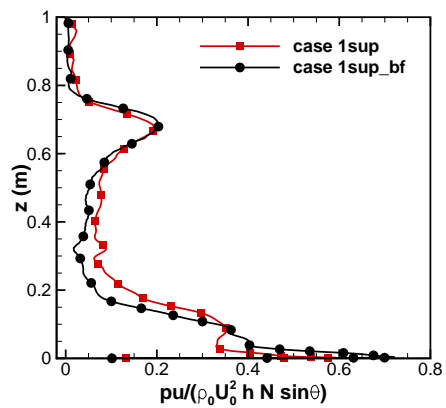

Figure 12: Normalized vertical profiles of the baroclinic flux (product of pressure and velocity amplitudes) for case (a) $1 \mathrm{sub}$, (b) $1 \mathrm{cri}$, (c) $1 \mathrm{sup}$, and (d) $2 \mathrm{cri}$. All the profiles are shown at $x / l=1.58$, a location away from the topography.

where $\phi=u_{i}, p$ and $\left\langle\phi>_{y T}(x, z)=\frac{1}{T} \int_{t}^{t+T}\left\langle\phi>_{y}(x, z, t) d t\right.\right.$ which is usually very small after the initial transient.

The baroclinic response is quantified by the analysis of the baroclinic energy budget. The equation for the baroclinic energy with diffusive fluxes of wave energy neglected and averaged over complete tidal cycle is given by (also see Eq. 4.1 of Rapaka et al. (2013) and Kang and Fringer (2011).).

$$
\frac{\Delta E}{T}+\nabla_{H} \cdot\left\langle\overline{\mathbf{F}}_{b c}+\overline{\mathbf{F}}_{a d v}\right\rangle_{T}=\langle\bar{C}\rangle_{T}-\left\langle\bar{\varepsilon}_{b c}\right\rangle_{T}-\langle\bar{P}\rangle_{T}
$$

where,

$$
\begin{gathered}
E=K E+P E, \quad K E=\frac{1}{2}\left(u_{b c}{ }^{2}+v_{b c}{ }^{2}+w_{b c}{ }^{2}\right), \quad P E=\frac{1}{2} N_{\infty}{ }^{-2}\left(g \frac{\rho^{*}}{\rho_{0}}\right)^{2} \\
\mathbf{F}_{b c}=p_{b c} \mathbf{u}_{\mathbf{b c}}, \quad \mathbf{F}_{a d v}=\mathbf{u} *\left(E+u_{b t} * u_{b c}\right), \quad C=\frac{\partial p^{*}}{\partial z} W(z), \quad W(z)=-\frac{\partial([z-h(x)] U)}{\partial x} \\
\varepsilon_{b c}=v \frac{\partial\left(u_{b c}\right)_{i}}{\partial x_{j}} \frac{\partial\left(u_{b c}\right)_{i}}{\partial x_{j}}, \quad P \equiv-\left\langle u_{i}^{\prime} u_{j}^{\prime}\right\rangle_{y}\left\langle S_{i j}\right\rangle_{y}-\left\langle\tau_{i j}\right\rangle_{y}\left\langle S_{i j}\right\rangle_{y} .
\end{gathered}
$$

The overbar represents depth integration. $\bar{C}$ is the conversion from the barotropic to the baroclinic wave field, $\bar{\varepsilon}_{b c}$ is the viscous dissipation of the baroclinic energy, $\overline{\mathbf{F}}_{b c}$ is the wave energy flux, $\overline{\mathbf{F}}_{a d v}$ is the advective energy flux, and $P$ is the turbulent production. The operator $\nabla_{H}$ is the horizontal gradient which simplifies to $\frac{\partial}{\partial x}$ in the present problem that has topography that is 2-dimensional in the mean. Note that $S_{i j}$ is the rate of strain tensor and $\tau_{i j}$ is the subgrid scale tensor which is non-zero only when LES is used. Physically, the topographic energy conversion (at rate $C$ ) from the oscillatory forcing is transported away from the obstacle as internal waves with a energy flux $\left(F_{b c}\right)$, and the rest of the terms in the energy balance act as a loss insofar as the internal wave energy field. Linear theory assumes that $C \equiv F_{b c}$. 


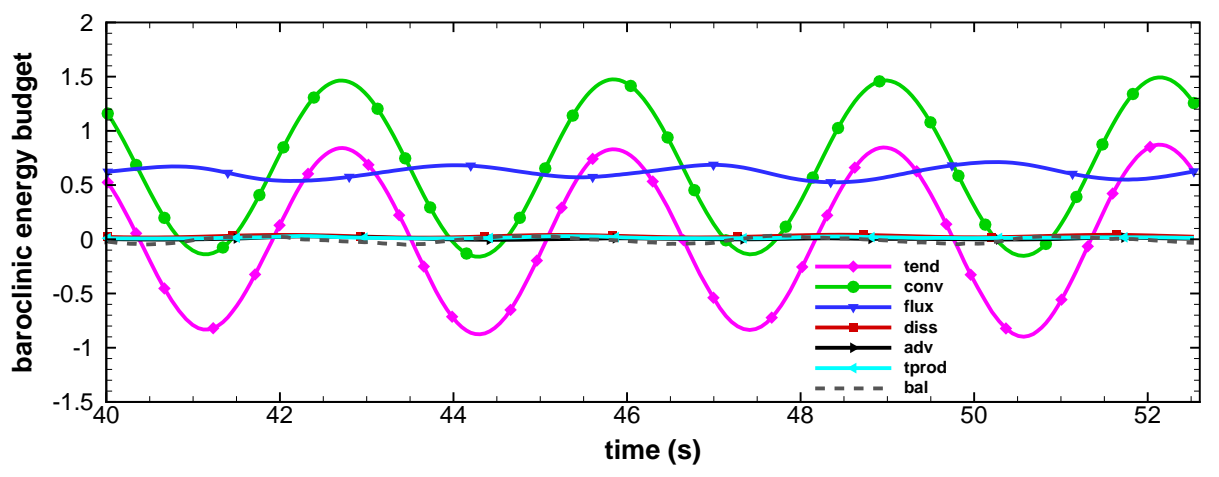

Figure 13: Evolution of various terms in the barolclinic energy budget, shown in table 5, integrated over an area from $x=-3 m$ to $x=+3 m$ for case 1 cri. The balance shown is the net difference between the baroclinic energy conversion and the sum of all other terms including tendency, wave flux, baroclinic dissipation, advection, and turbulence production.

Figure 12 shows the normalized vertical profile of the baroclinic wave flux amplitude at a location away from the topography $(x / l=-1.58)$. The amplitude of a variable is computed as its root mean square over two complete tidal cycles. The IBM results compare well with the previously published BFG results, especially in case 1 with $E x=0.066$. Fig. 12(a) compares the wave flux of case 1sub with case 1sub_bf . The region around the peak near $z=1.8 \mathrm{~m}$ indicates the location of the tidal beam where there is a local maximum in the baroclinic response. The agreement in Case 1sub using IBM is excellent with with case 1sub_bf that had employed a body fitted grid. As the criticality parameter increases, the tidal beam becomes shallower as shown in fig. 12(b) for case 1cri and fig. 12(c) for case 1 sup which show the location of the beam center around $z=1.2 \mathrm{~m}$ and $z=0.65 \mathrm{~m}$, respectively. The profile of the baroclinic wave flux for case 1cri is in good agreement with that for case 1cri_bf obtained using a body fitted grid (Rapaka et al., 2013). Figure 12(c) illustrates the baroclinic response for the supercritical case 1sup. The supercritical case has a thinner beam. The baroclinic intensification in case 1 sup is slightly larger compared to case $1 \mathrm{cri}$. The profiles show good agreement between IMB and BFG in the supercritical case too. However, in both critical and supercritical cases, the baroclinic response near the topography is slightly stronger.

Figure 12(d) shows the response for case 2cri with $E x=0.4$ which has a larger forcing amplitude relative to case 1 . Owing to large $E x$, the advective term cannot be neglected with respect to the temporal term in determining the wave response resulting in weaker beam-like behavior in case 2 cri. The baroclinic energy is also spread into higher harmonics which can be seen by the secondary peak in fig. 12(d). The baroclinic wave flux is larger in case 2cri compared to case 2cri_bf that utilizes a body fitted grid (Jalali et al., 2014). This is likely due to the lower resolution of the wave field in case 2 cri compared to case 2cri_bf . The distribution of points has to be more uniform near the boundary in the IBM case relative to the body-fitted grid in order to have reasonable resolution of wallnormal gradients at the surface of the obstacle. Therefore, the resolution of the wave field away from the boundary is necessarily less in IBM relative to BFG even if the number of grid points in the fluid region is approximately similar between IMB and BFG methods.

Figure 13 shows the evolution of various terms in the baroclinic energy budget integrated over an area from $x=-3 m$ to $x=+3 m$ enclosing the topography for case 1 cri. The conversion of energy from barotropic to baroclinic flow is balanced, to a large extent, by baroclinic wave flux and tendency terms. There is substantial oscillatory modulation in $C$ that leads to a modulation in the tendency term. To quantify the energetics, the area integrated baroclinic energy budget is averaged over two complete tidal cycles and shown in table 5 .

Table 5 shows the baroclinic energy budget including the conversion to internal waves $(C)$, radiated wave flux $(M$ or $\left.F_{b c}\right)$, advective flux $\left(F_{a d v}\right)$, the baroclinic energy dissipation $\left(\varepsilon_{b c}\right)$ and the turbulence production $(P)$ which appear in the baroclinic energy budget (see Eq. 49). For case 1sub, the resolution is sufficient and both the conversion and the baroclinic flux agree well with case 1sub_bf . For the critical case (resonant case of wave generation) there is some difference, e.g. the wave flux in case 1cri_bf is about $10 \%$ lower than the IBM results. For the supercritical case, the 


\begin{tabular}{rcccccccc} 
case & $E x$ & tendency & $C$ & $F_{b c}$ & $\varepsilon_{b c}$ & $F_{a d v}$ & $\mathrm{P}$ & balance \\
\hline 1sub & 0.066 & 0.000 & 0.689 & 0.691 & 0.019 & 0.000 & 0.002 & -0.022 \\
1cri & 0.066 & 0.005 & 0.665 & 0.617 & 0.029 & 0.006 & 0.016 & -0.006 \\
1sup & 0.066 & -0.005 & 1.009 & 0.962 & 0.021 & -0.007 & 0.017 & 0.020 \\
1sub_bf & 0.066 & 0.011 & 0.689 & 0.681 & 0.012 & 0.002 & 0.003 & -0.004 \\
1cri_bf & 0.066 & -0.008 & 0.572 & 0.551 & 0.013 & 0.002 & 0.025 & -0.011 \\
1 sup_bf & 0.066 & -0.008 & 0.843 & 0.824 & 0.007 & -0.007 & 0.028 & -0.008 \\
1 cri_ls & 0.066 & 0.009 & 0.704 & 0.645 & 0.078 & 0.007 & 0.017 & -0.052 \\
2cri & 0.400 & 0.013 & 0.726 & 0.609 & 0.075 & 0.081 & 0.025 & -0.077 \\
2cri_bf & 0.400 & 0.001 & 0.574 & 0.463 & 0.054 & 0.050 & 0.022 & -0.023 \\
2cri_ls & 0.400 & -0.003 & 0.721 & 0.612 & 0.087 & 0.092 & 0.031 & -0.098 \\
\hline
\end{tabular}

Table 5: Baroclinic energy budget: tendency $\left(\frac{\Delta E}{T}\right)$, energy conversion to waves $(C)$, radiated wave flux $\left(M \equiv F_{b c}\right)$, baroclinic energy dissipation $\left(\varepsilon_{b c}\right)$, baroclinic advective flux $\left(M_{a d v} \equiv F_{a d v}\right)$, and turbulence production $(P)$, integrated over the area between $x=-3$ to $+3 \mathrm{~m}$. All the quantities are normalized by $\frac{\pi}{4} \rho_{0} U_{0}^{2} h^{2} \sqrt{N^{2}-\Omega^{2}}$, the term appearing in linear analysis. The balance term, small compared to the conversion, is the net difference between the baroclinic energy conversion $(C)$ and the sum of all other terms including tendency, wave flux, baroclinic dissipation, advection, and turbulence production.

wave flux in case 1sup_bf is smaller by about $15 \%$ compared to the IBM case. Thus, there are moderate differences in the the radiated flux predictions of the IBM with respect to the body fitted grid results and these differences depend on the slope angle through the slope criticality parameter .

\subsection{Turbulence}

Figure 14 shows the spatial distribution of turbulent kinetic energy (TKE) for cases 1cri_bf , 1cri and 1cri_ls at phase of peak upslope flow at the left midslope $(x / l=-0.405)$. The turbulence generated at the ridge under these conditions is mainly due to density overturns and shear instability in the stratified oscillating flow adjacent to the ridge slope that, while occurring in the boundary flow, are not primarily associated with the wall shear (Rapaka et al., 2013). The IBM captures these mechanisms well. Figure 14(a) corresponds to case 1cri_bf and is adapted from figure 8(c) of Rapaka et al. (2013). It shows the inhomogeneous distribution of TKE including a patch of turbulence extending from the bottom that was generated due to density overturns when the flow reverses from downslope to upslope and is then advected upward. Figure 14(b) shows the spatial distribution of TKE for case 1cri which compares well with case 1cri_bf. However, some turbulent features very close to the bottom are not resolved as well in IBM due to the lower wall-normal resolution. For instance, the thin patch of TKE in the downslope attached boundary layer on the right side of the ridge in fig. 14(a) is absent in fig. 14(b).

Figure 15 shows the spatial distribution of TKE for cases 2cri_bf , 2cri and 2cri_ls at time 6T where T is tidal period. Case 2 has a high excursion, $E x=0.4$. Figure 15 (a) corresponds to case 2cri_bf and is adapted from figure 8(c) of Jalali et al. (2014). It corresponds to a phase of down-to-upslope barotropic flow reversal and shows a large vertical patch of turbulence extending from the bottom to approximately twice the topographic height, on the lee side of the topography. This a result of the breaking lee waves as described in Jalali et al. (2014). Figure 15(b) shows the spatial distribution of TKE for case 2 cri which also exhibits breaking lee waves similar to case 2cri_bf . The spatial distribution of TKE is similar between the two methods but the TKE magnitude is somewhat larger in case 2 cri compared to case 2cri_bf which is consistent with the larger conversion to baroclinic energy which was seen in the IBM case 2 cri .

\section{LES of stratified flow past a large scale model ridge}

Cases 1cri_ls and 2cri_ls, shown in tables 3 and 4 correspond to tidal flow over a large scale topography with same shape as shown in fig. 10 but with length increased by 1000 times in all three directions. As a result, the $R e_{s}$ in these two cases is much larger compared to the laboratory scale topography. Also, the disparity between the scale of 
(a)

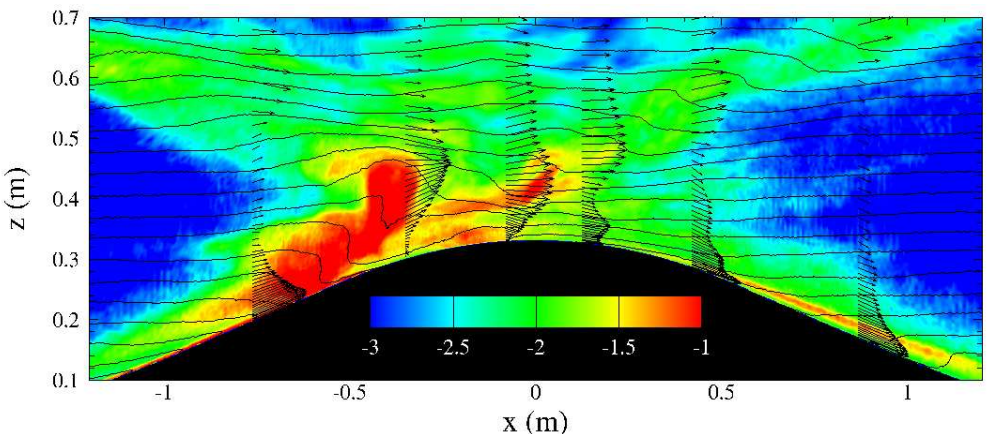

(b)

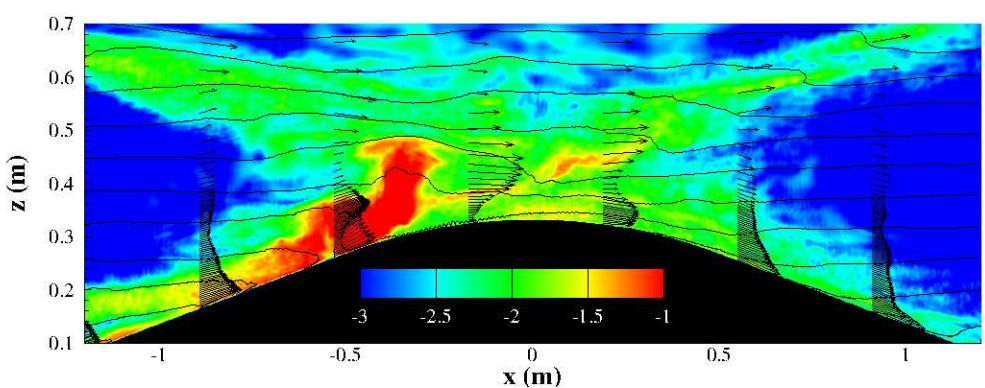

(c)

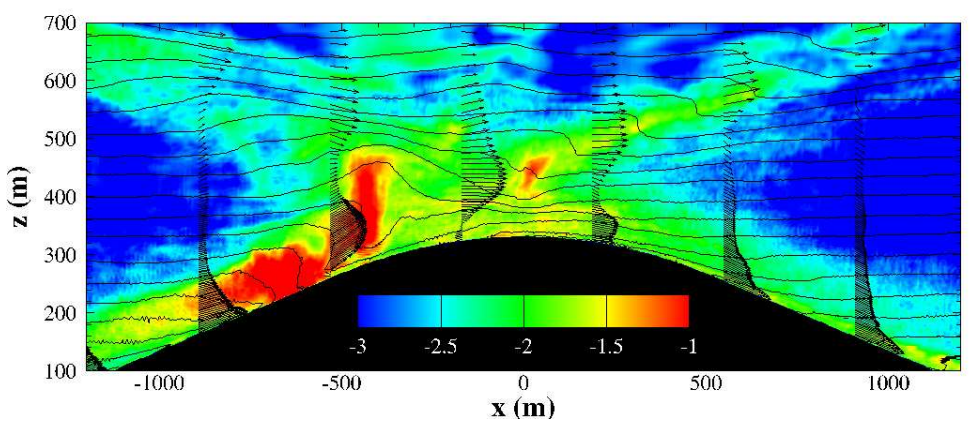

Figure 14: Spatial distribution of $\log _{10}\left(T K E / U_{0}^{2}\right)$ and isopycnals near the topography in (a) case 1cri_bf , (b) case 1cri, and (c) case 1cri_ls . Each snapshot corresponds to a phase of peak upslope flow at the left midslope $(x / l=-0.405)$. 
(a)

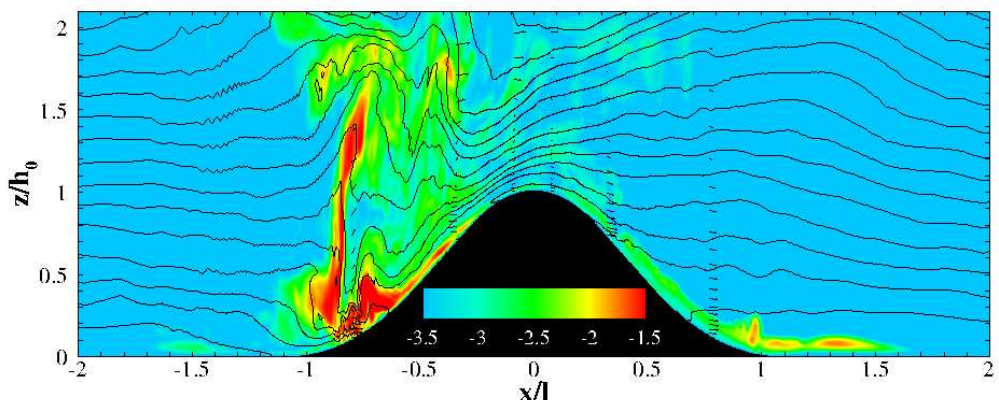

(b)

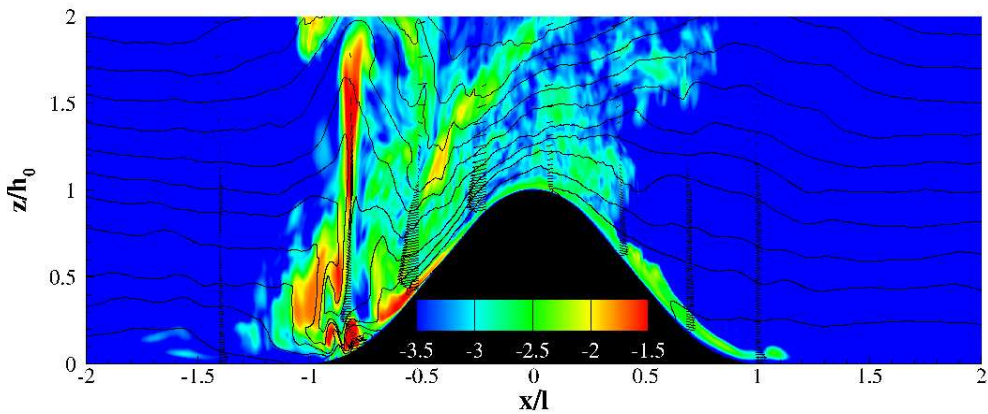

(c)

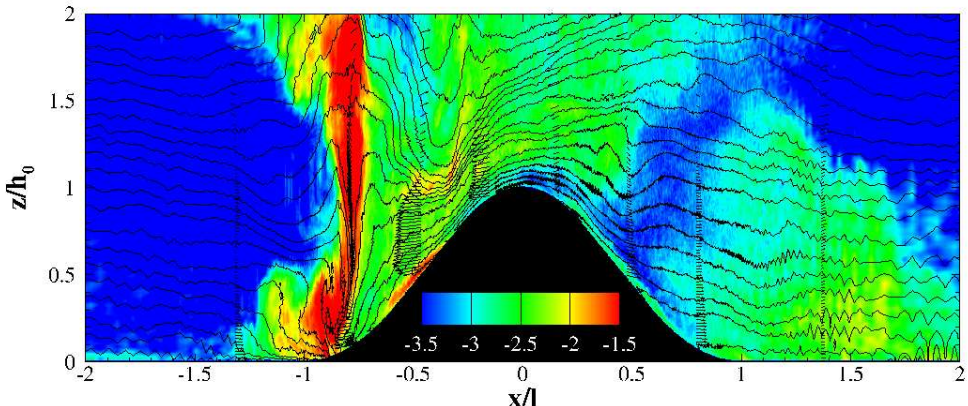

Figure 15: Spatial distribution of $\log _{10}\left(T K E / U_{0}^{2}\right)$ and isopycnals near the topography, shown at time=6T, in (a) case 2cri_bf , (b) case 2cri, and (c) case 2cri_ls. Note that $h_{0}$ is the height of the topography, and $T$ is the barotropic cycle time period. 
the large turbulent overturn $\left(2 h_{0}=600 \mathrm{~m}\right.$ in figure $\left.15(\mathrm{c})\right)$ and the viscous boundary layer increases for the large scale ridge. This makes DNS, which requires resolution of the turbulence dissipative length scales, prohibitively expensive. To reduce the computational expense, LES is used. To further reduce the computational cost, the near-wall turbulence is not resolved, instead a nonlinear drag law (Eq. 40) is used to parametrize the bottom friction as described in section 4. A drag coefficient of 0.0025 , commonly used in oceanographic applications, is used.

For case 1cri_ls, all the non-dimensional parameters, except the $R e_{s}$, are kept the same as in case 1 cri by decreasing the buoyancy frequency and forcing frequencies. Note that the case 1cri_ls does not resolve the bottom boundary layer and employs a nonlinear bottom drag. Yet, the position of the peak velocity in the beam, as shown in figure 11, is very close compared to that of the case 1cri_bf, while the magnitude of the velocity is slightly higher.

In figure 12(b), the larger-scale and higher Re case 1cri_ls with $E x=0.066$, exhibits a baroclinic response that has little difference with respect to the lab-scale case 1 cri. At a higher $E x=0.4$, the larger-scale case 2 cri_ls exhibits a baroclinic response that, as shown in figure 12(d), is similar that of case 2 cri but with peaks that have somewhat larger amplitude.

Figure 14(c) shows the spatial distribution of TKE for case 1cri_ls corresponding to the same phase as in figure 14(a) for case 1cri_bf. An approximately $100 \mathrm{~m}$ tall patch of turbulence extends above the bottom boundary on the left slope and is qualitatively similar to that in the laboratory scale cases 1cri_bf and 1cri shown in figure 14(a) and (b). Note that the characteristic thickness of the oscillating stratified boundary jet (approximately $100 \mathrm{~m}$ ) determines the vertical extent of the turbulence patch near the bottom boundary.

Figure 15(c) shows the spatial distribution of TKE for case 2cri_ls which also show the breaking lee waves similar to case $2 \mathrm{cri}$. The breaking lee waves show a tall patch of TKE extending to a height of $600 \mathrm{~m}$. The nondimensional vertical extent of twice the topographic height is the same for both laboratory and large-scale problems with the same Ex.

\section{Conclusions}

A sharp interface Immersed Boundary Method is developed to simulate stratified turbulent flows over complex topography using a Cartesian grid. In the IBM, fluxes are computed at the centroids of the cut cells faces in the fluid region to improve mass conservation properties of the solver. Ghost cells are placed on both the fluid and solid sides of the immersed boundary depending on their proximity to the immersed boundary to enhance the numerical stability. DNS is used for laboratory scale simulations that have enough grid resolution to resolve the skin friction and other boundary layer properties. For large scale topographies with high Reynolds number and marginal boundary layer resolution, LES is chosen with the option of imposing a non-linear drag model near the solid walls. Various LES models including Standard and Dynamic Smagorinsky Models are made available in the solver.

The IBM solver developed here has been validated in several benchmark problems including flow past a twodimensional body (circular cylinder) and a three-dimensional body (sphere) in both steady and unsteady laminar flow regimes. The IBM method has second-order convergence as demonstrated in flow past a cylinder placed in channel. In the case of turbulent channel flow, mean and turbulence statistics agree well with prior results in both unstratified and stratified configurations.

The DNS approach has been used to evaluate the present IBM for tidal flow over a laboratory-scale (order of few meters) smoothed triangular ridge. The baroclinic energy budget and turbulence statistics have been examined at low $(E x=0.066)$ and high $(E x=0.4)$ forcing amplitudes under near critical flow conditions. In addition, for $E x=0.066$, the criticality parameter $(\varepsilon)$ is varied to evaluate the performance in subcritical and supercritical cases. The energy conversion and baroclinic wave flux are in excellent agreement with those obtained using a body fitted grid for the subcritical case. For critical and supercritical flows at $E x=0.066$, both the conversion and wave fluxes in the body fitted grid (BFG) are higher by approximately 10-15\% in the IBM case. The BFG results for conversion and wave flux in the critical slope case with large $E x=0.4$ (the case with direct breaking of lee waves) is also higher than the IBM, by about $20 \%$. We have thus been able to quantify the deviation of the IBM with respect to the more accurate BFG results over a wide dynamic range of wave generation and conclude that the deviation increases with increasing nonlinearity of the internal wave field in the generation region. The difference can be attributed to the fluid cells having a lower and more non-uniform resolution of the wave field in the IBM case relative to the BFG.

The phasing of turbulence statistics and spatial distribution of TKE field are studied. The mechanisms of turbulence generation, including the large convective overturns after the down-to-up flow reversal and the TKE generated 
due to shear production during the peak down-slope flow, are observed as described in Rapaka et al. (2013) and Jalali et al. (2014) using a body fitted grid.

LES are performed on a large scale topography, of the order of few kilometers, with the same Ex and the criticality parameter as in the small scale topography but significantly larger Reynolds number. Non-linear drag is imposed to parameterize the bottom friction. The intensification of the beam is slightly higher and so are the conversion and baroclinic wave flux. Phasing of turbulence is qualitatively similar when compared to the small scale topography.

IBM provides a viable approach to simulate flows over complex immersed bodies using Cartesian grids in a stratified environment as demonstrated here. With future work, it can also provide a powerful platform to be used with local grid refinement techniques (such as those used in adaptive mesh refinement methods) that are computationally economical. However, IBM also has limitations. It does not provide as high a resolution of wall-normal gradients as BFG. Since, boundary layer resolution and skin friction are not as important in most oceanic problems as in engineering applications, that inaccuracy of IBM is less of a concern. It is worth noting that the IBM method, for the same number of grid points as BFG, does not provide the same resolution of nonlinear flow features, e.g., the nonlinear aspects of the internal wave field studied in the present work. Therefore, whenever possible, the IBM method should be validated in the flow problem of interest.

\section{Acknowledgements}

We gratefully acknowledge support provided by NSF grant OCE-1459774, program manager Eric Itsweire. We are also pleased to acknowledge Vincenzo Armenio and Frederico Roman who provided a IBM preprocessor code that was modified and integrated into the solver developed here.

\section{Appendix A. Temporal integration}

The temporal integration is accomplished with a combination of RK3-ADI scheme. The explicit part is treated using explicit 3rd order Runge-Kutta method of Williamson (1980) and the implicit part is treated using an unconditionally stable ADI method in three dimensions with second-order accuracy in both time and space, see Douglas Jr. (1962) or page 934 of Pozrikidis (2008). The governing equations are given below,

$$
\begin{aligned}
\frac{\partial u_{i}}{\partial t} & =-\frac{\delta U_{k} u_{i}}{\delta x_{k}}-g \frac{\rho^{*}}{\rho_{0}} \delta_{i 3}+\frac{\delta}{\delta x_{k}}\left(v_{e} \frac{\delta u_{i}}{\delta x_{k}}\right)+\frac{\delta}{\delta x_{k}}\left(v_{e} \frac{\delta u_{k}}{\delta x_{i}}\right)-\frac{1}{\rho_{0}} \frac{\delta p}{\delta x_{i}} \\
& =E\left(u_{i}, \rho^{*}\right)+I\left(u_{i}, p\right)
\end{aligned}
$$

where $E\left(u_{i}, \rho^{*}\right)$ and $I\left(u_{i}, p\right)$ represent the explicit and implicit parts of the RHS of Eq. A.1 defined by,

$$
\begin{aligned}
E\left(u_{i}, \rho^{*}\right) & \equiv-\frac{\delta U_{k} u_{i}}{\delta x_{k}}-g \frac{\rho^{*}}{\rho_{0}} \delta_{i 3}+\frac{\delta}{\delta x_{k}}\left(v_{e} \frac{\delta u_{k}}{\delta x_{i}}\right) \\
I\left(u_{i}, p\right) & \equiv \frac{\delta}{\delta x_{k}}\left(v_{e} \frac{\delta u_{i}}{\delta x_{k}}\right)-\frac{1}{\rho_{0}} \frac{\delta p}{\delta x_{i}}
\end{aligned}
$$

Note that the cross terms arising from $v_{e}$ in LES are added to the right hand side of Eq. A.3. For DNS, these terms disappear due to continuity and $v_{e}$ is replaced by the molecular viscosity in the diffusion terms. The temporal integration can be demonstrated using a model ordinary differential equation,

$$
\dot{\Phi}=E(\Phi)+I(\Phi)
$$

where $E$ and $I$ include explicit and implicit terms respectively.

$$
\begin{aligned}
q^{m} & =C_{1}^{m} q^{m-1}+\Delta t E\left(\Phi^{m-1}\right) \\
\Phi^{m} & =\Phi^{m-1}+C_{2}^{m} q^{m}+C_{3}^{m} \Delta t I\left(\Phi^{m}\right)
\end{aligned}
$$


Table A.6: Coefficients of mixed RK3-ADI Scheme

\begin{tabular}{cccc}
\hline$m$ & $C_{1}$ & $C_{2}$ & $C_{3}$ \\
\hline 1 & 0 & $1 / 3$ & $1 / 3$ \\
2 & $-5 / 9$ & $15 / 16$ & $5 / 12$ \\
3 & $-153 / 128$ & $8 / 15$ & $1 / 4$ \\
\hline
\end{tabular}

Define $\Delta t_{r k^{m}} \equiv C_{3}^{m} \Delta t$, above equation can be written as,

$$
\Phi^{m}=\Phi^{m-1}+C_{2}^{m} q^{m}+\Delta t_{r k^{m}} I\left(\Phi^{m}\right)
$$

Note that above equation is implicit in $\Phi^{m}$, hence involves solving a system of linear equations.

The momentum equations A.2 can be marched using above method. The details are given below, Temporal integration of the momentum equations along with the scalar equations is performed from step $n$ to $n+1$ in three sub-steps. The equation for the density follows similar procedure and is not included here.

$$
\begin{aligned}
q^{1} & =\Delta t E\left(u_{i}^{n}, \rho^{\prime n}\right) \\
u_{i}^{1} & =u_{i}^{n}+C_{2}^{1} q^{1}+\Delta t_{r k^{1}} I\left(u_{i}^{1}, p^{1}\right) \\
q^{2} & =C_{1}^{2} q^{1}+\Delta t E\left(u_{i}^{1}, \rho^{\prime}\right) \\
u_{i}^{2} & =u_{i}^{1}+C_{2}^{2} q^{2}+\Delta t_{r k^{2}} I\left(u_{i}^{2}, p^{2}\right) \\
q^{3} & =C_{1}^{3} q^{2}+\Delta t E\left(u_{i}^{2}, \rho^{\prime 2}\right) \\
u_{i}^{n+1} & =u_{i}^{2}+C_{2}^{3} q^{3}+\Delta t_{r k^{3}} I\left(u_{i}^{n+1}, p^{n+1}\right)
\end{aligned}
$$

Appendix A.1. RK3-ADI step 1

This step includes RK3 sub-step 1 and simultaneous time integration of implicit terms using ADI method.

$$
\begin{aligned}
& q^{1}=\Delta t\left[-\frac{\delta U_{k}^{n} u_{i}^{n}}{\delta x_{k}}-g \frac{\rho^{* n}}{\rho_{0}} \delta_{i 3}+\frac{\delta}{\delta x_{k}}\left(v_{e} \frac{\delta u_{k}^{n}}{\delta x_{i}}\right)\right] \\
& u_{i}^{1}=u_{i}^{n}+C_{2}^{1} q^{1}+\Delta t_{r k^{1}} \frac{\delta}{\delta x_{k}}\left(v_{e} \frac{\delta u_{i}^{1}}{\delta x_{k}}\right)-\frac{\Delta t_{r k^{1}}}{\rho_{0}} \frac{\delta p^{1}}{\delta x_{i}}
\end{aligned}
$$

After re-arranging above equation, we have:

$$
\left[1-\Delta t_{r k^{1}} \frac{\delta}{\delta x_{k}}\left(v_{e} \frac{\delta}{\delta x_{k}}\right)\right] u_{i}^{1}=u_{i}^{n}+C_{2}^{1} q^{1}-\frac{\Delta t_{r k^{1}}}{\rho_{0}} \frac{\delta p^{1}}{\delta x_{i}}
$$

Details of the predictor-corrector algorithm and treatment of flow variables on a collocated grid arrangement are discussed separately in section 2 . The equations solved are briefly presented below.

\section{Appendix A.1.1. Predictor step}

Let $p^{1}=p^{*}+p^{\prime}$ and $u_{i}^{1}=u_{i}^{*}+u_{i}^{\prime}$, and use $p^{*}=p^{n}$

$$
\left[1-\Delta t_{r k^{1}} \frac{\delta}{\delta x_{k}}\left(v_{e} \frac{\delta}{\delta x_{k}}\right)\right] u_{i}^{*}=u_{i}^{n}+C_{2}^{1} q^{1}-\frac{\Delta t_{r k^{1}}}{\rho_{0}} \frac{\delta p^{n}}{\delta x_{i}}
$$

Appendix A.1.2. ADI method in 3D

ADI method of Douglas (1962) which is unconditionally stable and second order accurate in time and space is used here to solve Eq. A.18. Let us denote the right hand side by $S \equiv u_{i}^{n}+C_{2}^{1} q^{1}-\frac{\Delta t_{r k}}{\rho_{0}} \frac{\delta p^{n}}{\delta x_{i}}$. The solution is then marched from $u_{i}^{n}$ to $u_{i}^{*}$ in three steps

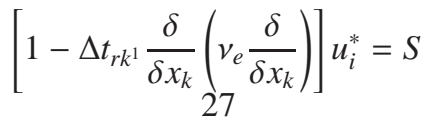


ADI step 1

$$
\left[1-\frac{\Delta t_{r k^{1}}}{2} \frac{\delta}{\delta x}\left(v_{e} \frac{\delta}{\delta x}\right)\right] u_{i}^{\dagger}=S+\Delta t_{r k^{1}}\left[\frac{1}{2} \frac{\delta}{\delta x}\left(v_{e} \frac{\delta}{\delta x}\right)+\frac{\delta}{\delta y}\left(v_{e} \frac{\delta}{\delta y}\right)+\frac{\delta}{\delta z}\left(v_{e} \frac{\delta}{\delta z}\right)\right] u_{i}^{n}
$$

ADI step 2

$$
\left[1-\frac{\Delta t_{r k^{1}}}{2} \frac{\delta}{\delta y}\left(v_{e} \frac{\delta}{\delta y}\right)\right] u_{i}^{\ddagger}=u_{i}^{\dagger}-\frac{\Delta t_{r k^{1}}}{2} \frac{\delta}{\delta y}\left(v_{e} \frac{\delta u_{i}^{n}}{\delta y}\right)
$$

ADI step 3

$$
\left[1-\frac{\Delta t_{r k^{1}}}{2} \frac{\delta}{\delta z}\left(v_{e} \frac{\delta}{\delta z}\right)\right] u_{i}^{*}=u_{i}^{\ddagger}-\frac{\Delta t_{r k^{1}}}{2} \frac{\delta}{\delta z}\left(v_{e} \frac{\delta u_{i}^{n}}{\delta z}\right)
$$

Appendix A.1.3. Poisson equation

$$
\frac{\delta}{\delta x_{k}} \frac{\delta p^{\prime}}{\delta x_{k}}=\frac{\rho_{0}}{\Delta t_{r k^{1}}} \frac{\delta U_{i}^{*}}{\delta x_{i}}
$$

Note that $U_{i}^{*}$ is obtained by interpolating $u_{i}^{*}$ onto the cell face and adding a modified pressure-gradient as described in section 2 .

Appendix A.1.4. Corrector step

$$
\begin{aligned}
p^{1} & =p^{*}+p^{\prime} \\
u_{i}^{1} & =u_{i}^{*}-\Delta t_{r k^{1}} \frac{\delta p^{\prime}}{\delta x_{i}} \\
U_{i}^{1} & =U_{i}^{*}-\Delta t_{r k^{1}} \frac{\delta p^{\prime}}{\delta x_{i}}
\end{aligned}
$$

The time marching of RK3-ADI sub-steps 2 and 3 follow a similar procedure and are not discussed here.

\section{References}

Alford, M. H., MacKinnon, J. A., Nash, J. D., Simmons, H., Pickering, A., Klymak, J. M., Pinkel, R., Sun, O., Rainville, L., Musgrave, R., Beitzel, T., Fu, K.-H., Lu, C.-W., 2011. Energy flux and dissipation in Luzon strait: Two tales of two ridges. J. Phys. Oceanogr. 41, 2211-2222. Armenio, V., Sarkar, S., 2002. An investigation of stably stratified turbulent channel flow using large-eddy simulation. J. Fluid Mech. 459 , 1-42. Arthur, R. S., Fringer, O. B., 2014. The dynamics of breaking internal solitary waves on slopes. J. Fluid Mech. 761, 360-398.

Balaras, E., 2004. Modeling complex boundaries using an external force field on fixed cartesian grids in large-eddy simulations. Comp. Fluids 33, 375-404.

Chalamalla, V. K., Gayen, B., Scotti, A., Sarkar, S., 2013. Turbulence during the reflection 0of internal graity waves at critical and near-critical slopes. J. Fluid Mech. 729, 47-68.

Deardorff, J. W., 1970. A numerical study of three-dimensional turbulent channel flow at large Reynolds numbers. J. Fluid Mech. $41,453$.

Douglas Jr., J., 1962. Alternating direction methods for three space variables. Numerische Mathematik 4, 41-63.

Fadlun, E. A., Verzicco, R., Orlandi, P., Mohd-Yusof, J., 2000. Combined immersed-boundary finite difference methods for three-dimensional complex flow simulations. J. Comp. Phy. 161, 35-60.

Fringer, O. B., Gerritsen, M., Street, R. L., 2006. An unstructured-grid, finite-volume, nonhydrostatic, parallel coastal ocean simulator. Ocean Modelling 14 (3-4), 139-173.

Garcia-Villalba, M., del Álamo, J. C., 2011. Turbulence modification by stable stratification in channel flow. Phy. Fluids $23,045104$. Gayen, B., Sarkar, S., 2011a. Boundary mixing by density overturns in an internal tidal beam. Geophys. Res. Lett. 38 , L14608.

Gayen, B., Sarkar, S., 2011b. Direct and large eddy simulations of internal tide generation at a near critical slope. J. Fluid Mech. 681, 48-79. Germano, M., Piomelli, U., Moin, P., Cabot, W. H., 1991. A dynamic subgrid-scale eddy viscosity model. Phys. Fluids 3(7), 1760-1765. Henderson, R. D., 1995. Details of the drag curve near the onset of vortex shedding. Phy. Fluids 7, 2102-2104. 
Jalali, M., Rapaka, N. R., Sarkar, S., 2014. Tidal flow over topography: effect of excursion number on wave energetics and turbulence. J. Fluid Mech. 750, 259-283.

Johnson, T. A., Patel, V. C., 1999. Flow past a sphere up to a Reynolds number of 300. J. Fluid Mech. 378, 19-70.

Kang, D., Fringer, O., 2011. Energetics of barotropic and baroclinic tides in the monterey bay area. J. Phys. Oceanogr. 42, $272-290$.

Kang, S., Iaccarino, G., Moin, P., 2009. Accurate immersed-boundary reconstructions for viscous flow simulations. AIAA Journal 47, 1750.

Klymak, J. M., Legg, S., Pinkel, R., 2010. A simple parameterization of turbulent tidal mixing near supercritical topography. J. Phys. Oceanogr. 40, 2059-2074.

Klymak, J. M., Moum, J. N., Nash, J. D., Kunze, E., Girton, J. B., Carter, G. S., Lee, C. M., Sanford, T. B., Gregg, M. C., 2006. An estimate of tidal energy lost to turbulence at the Hawaiian ridge. J. Phys. Oceanogr. 36, 1148-1164.

Klymak, J. M., Pinkel, R., Rainville, L., 2008. Direct breaking of the internal tide near topography: Kaena ridge, Hawaii. J. Phys. Oceanogr. 38, 380-399.

Lamb, K. G., 2014. Internal wave breaking and dissipation mechanisms on the continental slope/shelf. Ann. Rev. Flu. Mech. 46, $231-254$.

Ledwell, J. R., Montgomery, K. L., Polzin, K. L., St. Laurent, L. C., Schmitt, R. W., Toole, J. M., 2000. Evidence for enhanced mixing over rough topography in the abyssal ocean. Nature 403, 179-182.

Legg, S., Hallberg, R. W., Girton, J. B., 2006. Comparison of entrainment in overflows simulated by z-coordinate, isopycnal and non-hydrostatic models. Ocean Modelling 11, 69-97.

Legg, S., Klymak, J., 2008. Internal hydraulic jumps and overturning generated by tidal flows over a tall steep ridge. J. Phys. Oceanogr. 38, 1949-1964.

Llewellyn Smith, S. G., Young, W. R., 2002. Conversion of the barotropic tide. J. Phys. Oceanogr. 32, $1554-1566$.

Mark, A., van Wachem, B. G. M., 2008. Derivation and validation of a novel implicit second-order accurate immersed boundary method. J. Comp. Phy. 227, 6660-6680.

Marshall, J., Adcroft, A., Hill, C., Perelman, L., Heisey, C., 1997. A finite-volume, incompressible navier stokes model for studies of the ocean on parallel computers. J. Geophysical Res. 102, 5753-5766.

Meyer, M., Devesa, A., Hickel, S., Hu, X. Y., Adams, N., 2010. A conservative immersed interface method for large-eddy simulation of incompressible flows. J. Comp. Phy. 229, 6300-6317.

Mittal, R., Dong, H., Bozkurttas, M., Najjar, F. M., Vargas, A., von Loebbecke, A., 2008. A versatile sharp interface immersed boundary method for incompressible flows with complex boundaries. J. Comp. Phy. 227, 4825-4852.

Mittal, R., Iaccarino, G., 2005. Immersed boundary methods. Ann. Rev. Flu. Mech. 37, 239-261.

Moser, R. D., Kim, J., Mansour, N. N., 1999. Direct numerical simulation of turbulent channel flow up to Re $_{\tau}=590$. Phy. Fluids 11 , $943-945$.

Moum, J. N., Caldwell, D. R., Nash, J. D., Gunderson, G. D., 2002. Observations of boundary mixing over the continental slope. J. Phys. Oceanogr. 32, 2113-2130.

Nash, J. D., Alford, M. H., Kunze, E., Martini, K., Kelly, S., 2007. Hotspots of deep ocean mixing on the Oregon continental slope. Geophys. Res. Lett. 34, L01605.

Pétrélis, F., Llewellyn Smith, S. G., Young, W. R., 2006. Tidal conversion at submarine ridge. J. Phys. Oceanogr. 36, 1053-1071.

Pozrikidis, C., 2008. Numerical Computation in Science and Engineering, 2nd Edition. Oxford University Press.

Rapaka, N. R., Gayen, B., Sarkar, S., 2013. Tidal conversion and turbulence at a model ridge: direct and large eddy simulations. J. Fluid Mech. $715,181-209$.

Rhie, C. M., Chow, W. L., 1983. Numerical study of the turbulent flow past an airfoil with trailing edge separation. AIAA J. 21.

Rogallo, R. S., 1981. Numerical experiments in homogeneous turbulence. NASA Tech. Rep. TM-81315.

Roman, F., Napoli, E., Milici, B., Armenio, V., 2009. An improved immersed boundary method for curvilinear grids. Compters \& Fluids 38, $1510-1527$.

Santilli, E., Scotti, A., 2015. The stratified ocean model with adaptive refinement (somar). J. Comp. Phy. 291, 60-81.

Seo, J. H., Mittal, R., 2011. A sharp-interface immersed boundary method with improved mass conservation and reduced spurious pressure oscillations. J. Comp. Phy. 230, 7347-7363.

St. Laurent, L. C., Toole, J. M., Schmitt, R. W., 2001. Buoyancy forcing by turbulence above rough topography in the abyssal Brazil Basin. J. Phys. Oceanogr. 31, 3476-3495.

Taylor, J. R., Sarkar, S., 2008. Stratification effects in a bottom Ekman layer. J. Phys. Oceanogr. 38 (11), $2535-2555$.

Udaykumar, H. S., Mittal, R., Rampunggoon, P., Khanna, A., 2001. A sharp interface cartesian grid method for simulating flows with complex moving boundaries. J. Comp. Phy. 174, 345-380.

Venayagamoorthy, S. K., Fringer, O. B., 2007. On the formation and propagation of nonlinear internal boluses across a shelf break. J. Fluid Mech. 577, 137-159.

Venayagamoorthy, S. K., Stretch, D. D., 2010. On the turbulent prandtl number in homogeneous stably stratified turbulence. J. Fluid Mech. 644, 359-369.

Wunsch, C., Ferrari, R., 2004. Vertical mixing, energy, and the general circulation of the oceans. Annual Review of Fluid Mechanics 36, $281-314$.

Ye, T., Mittal, R., Udaykumar, H. S., Shyy, W., 1999. An accurate cartesian grid method for viscous incompressible flows with complex immersed boundaries. J. Comp. Phy. 156, 209-240.

Zang, Y., Street, R. L., Koseff, J. R., 1994. A non-staggered grid, fractional step method for time-dependent incompressible navier-stokes equations in curvilinear coordinates. J. Comp. Phy. 114, 18-33.

Zhang, Z., Fringer, O. B., Ramp, S. R., 2011. Three-dimensional, nonhydrostatic numerical simulation of nonlinear internal wave generation and propagation in the South China Sea. J. Geophys. Res. 116, C05022. 